\title{
SYNTHESIS OF 4-THIAZOLIDINE DERIVATIVES OF 6-NITROINDAZOLE: PHARMACEUTICAL IMPORTANCE
}

\author{
PUSHKAL SAMADHIYA*, RITU SHARMA, SANTOSH K. SRIVASTAVA, SAVITRI D. SRIVASTAVA \\ Department of Chemistry, Dr. H.S. Gour University (A Central University), Sagar-470003, India \\ (Received: August 2, 2011 - Accepted: December 26, 2011)
}

\begin{abstract}
New series of $\mathrm{N}$-[3-(1H-6-nitroindazol-1-yl)-propyl]-2-(substituted phenyl)-4-oxo-5-(substituted benzylidene)-1,3-thiazolidine-carboxamide, compounds $\mathbf{5}(\mathrm{a}-\mathrm{j})$ have been synthesized from 6-nitroindazole. Structures of all the synthesized compounds were confirmed by chemical and spectral analyses such as IR, ${ }^{1} \mathrm{H}$ NMR, ${ }^{13} \mathrm{C}$ NMR and FAB-Mass. All the synthesized compounds were screened for their antibacterial, antifungal, antitubercular and antiinflammatory activities.
\end{abstract}

Keyword: 6-nitroindazole, thiazolidinone, antimicrobial, antitubercular, antiinflammatory.

\section{INTRODUCTION}

Several azoles scaffold have been described in literature including indazole. Indazole derivatives are important nitrogen containing nine membered bicyclic heterocyclic compounds with applications as several biological activities and agrochemicals besides possessing important pharmacological activities such as antimicrobial, protein kinase inhibitors, antiproliferative, nitric oxide synthesis, anesthesia and antiprotozoal activity ${ }^{1-6}$. The indazole ring system is also present in many other compounds such as herbicides, dyes or sweeteners like guanidine- $1 H$-indazole. Despite the many useful applications of indazole derivatives, indazole chemistry remains less studied compared to other heteroaromatic compounds such as indole or benzimidazole. Indazole is a ten- $\pi$ electron aromatic heterocyclic system. Like the pyrazole molecule, indazole resembles both pyridine and pyrrole and its reactivity reflects this dual behaviour.

Thiazolidine ring system derives special important from the fact that it plays important role in medicinal chemistry. Substituted thiazolidine derivatives represent important key intermediates for synthesis of pharmacologically active drug thiazolidinone has wide range of biological activities such as antifungal, antiproliferative, antiinflammatory, antimalarial, herbicidal and antiviral property ${ }^{7-12}$.

As part of a continuing effort to develop novel heterocyclic compounds with potential therapeutic biological activities, several chemists are currently involved in the synthesis of numbers of indazole derivatives. In the present study, we have decided to synthesize new series of compounds showed in Scheme 1.

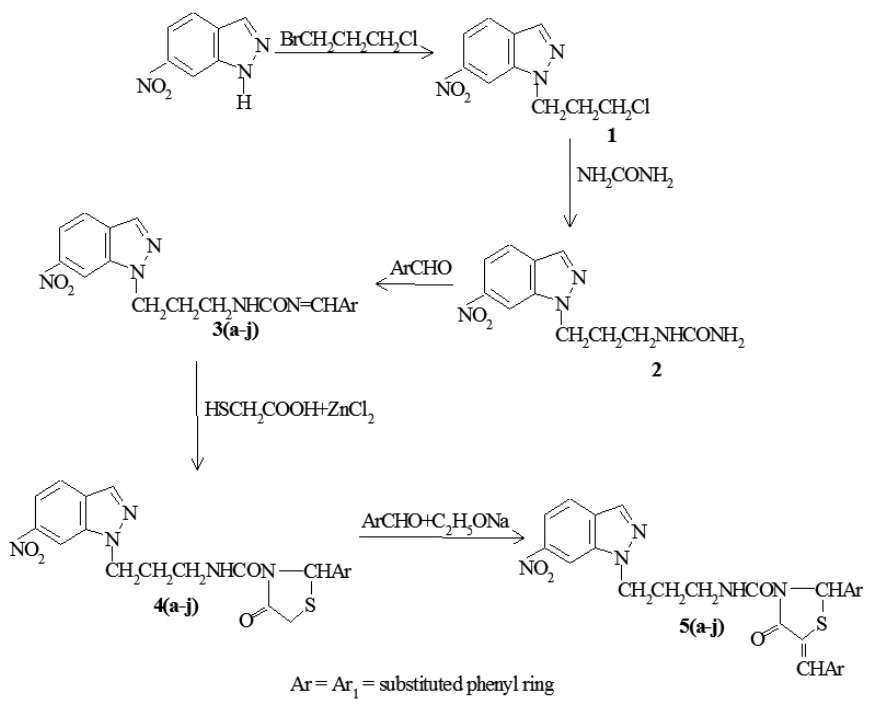

Scheme 1: Synthesis of compounds 1, 2, 3(a-j), 4(a-j) and 5(a-j).

\section{EXPERIMENTAL}

Melting points were taken in open capillaries and are uncorrected. Progress of reaction was monitored by silica gel-G coated TLC plates in $\mathrm{MeOH}: \mathrm{CHCl}_{3}$ system (1:9). The spot was visualized by exposing dry plate in iodine vapours. IR spectra were recorded in $\mathrm{KBr}$ disc on a Schimadzu 8201 PC, FTIR spectrophotometer $\left(\mathrm{n}_{\max }\right.$ in $\left.\mathrm{cm}^{-1}\right)$ and ${ }^{1} \mathrm{H}$ and ${ }^{13} \mathrm{C}$ NMR spectra were measured on a Brucker DRX-300 spectrometer in $\mathrm{CDCl}_{3}$ at 300 and $75 \mathrm{MHz}$ respectively using TMS as an internal standard. All chemical shifts were reported on d scales. The FAB-Mass spectra were recorded on a Jeol SX-102 mass spectrometer. Elemental analyses were performed on a Carlo Erba-1108 analyzer. The analytical data of all the compounds were highly satisfactory. For column chromatographic purification of the products, Merck silica Gel 60 (230-400 Mesh) was used. Inflammatory (in vivo) study has been approved by institutional ethical committee, Dr. H.S. Gour University, Sagar. The reagent grade chemicals were purchased from the commercial sources and further purified before use.

Method for the synthesis of the compound 1:

6-Nitroindazole $(0.308$ mole $)$ and 1-bromo-3-chloropropane $(0.308$ mole $)$ in ethanol $(100 \mathrm{ml})$ were stirred on a magnetic stirrer for about 6.30 hours at room temperature. The completion of the reaction was monitored by silica gel-G coated TLC plates. After the completion of the reaction the product was filtered and purified over a silica gel packed column chromatography using $\mathrm{CHCl}_{3}: \mathrm{CH}_{3} \mathrm{OH}(8: 2 \mathrm{v} / \mathrm{v})$ system as eluant $(150 \mathrm{ml})$. The purified product was dried under vacuo and recrystallized from acetone at room temperature to yield compound 1 (Figure 1).<smiles>O=[N+]([O-])c1ccc2cnn(CCCCl)c2c1</smiles>

Figure 1: Structure of compound 1.

$\mathbf{N}^{1}$-(3-chloropropyl)-6-nitroindazole 1:

Yield: $65 \%$; m.p. $163-165^{\circ} \mathrm{C}$; IR $\left(\mathrm{cm}^{-1}\right)$ : $768(\mathrm{C}-\mathrm{Cl}), 899(\mathrm{C}-\mathrm{N}), 1326(\mathrm{~N}-$ $\left.\mathrm{CH}_{2}\right), 1532\left(\mathrm{NO}_{2}\right), 1572(\mathrm{C}=\mathrm{C}), 1448,2842,2889,\left(\mathrm{CH}_{2}\right), 3020(\mathrm{CH}-\mathrm{Ar}) ;{ }^{1} \mathrm{H}$ NMR ( $\left.\mathrm{CDCl}_{3}, 300 \mathrm{MHz}\right) \delta: 2.20-2.26(\mathrm{~m}, 2 \mathrm{H}, \mathrm{H}-9), 3.41(\mathrm{t}, 2 \mathrm{H}, J=7.45 \mathrm{H}-10)$, $4.26(\mathrm{t}, 2 \mathrm{H}, J=7.45 \mathrm{~Hz}, \mathrm{H}-8), 7.86-8.35(\mathrm{~m}, 4 \mathrm{H}, \mathrm{Ar}-\mathrm{H}) ;{ }^{13} \mathrm{C}$ NMR $\left(\mathrm{CDCl}_{3}, 75\right.$

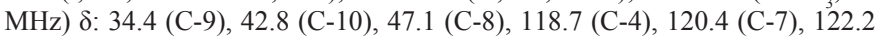
(C-5), 126.1 (C-3a), 136.2 (C-6), 137.0 (C-3), 135.7 (C-7a),; FAB-Mass $(\mathrm{m} / \mathrm{z})$ : 239 [M+]; Anal. Calcd. for $\mathrm{C}_{10} \mathrm{H}_{10} \mathrm{~N}_{3} \mathrm{O}_{2} \mathrm{Cl}$ : C 50.20, $\mathrm{H} 4.20, \mathrm{~N}$ 17.11; found $\mathrm{C}$ 50.17, H 4.13, N, 17.08.

Method for the synthesis of the compound 2:

Compound $1(0.208 \mathrm{~mole})$ and urea $(0.208 \mathrm{~mole})$ in ethanol $(100 \mathrm{ml})$ were stirred on a magnetic stirrer for about 4.30 hours at room temperature. The completion of the reaction was monitored by silica gel-G coated TLC plates. After the completion of the reaction the product was filtered and purified over a silica gel packed column chromatography using $\mathrm{CHCl}_{3}: \mathrm{CH}_{3} \mathrm{OH}(8: 2 \mathrm{v} / \mathrm{v})$ 
system as eluant $(120 \mathrm{ml})$. The purified product was dried under vacuo and recrystallized from ethanol at room temperature to yield compound $\mathbf{2}$ (Figure 2).

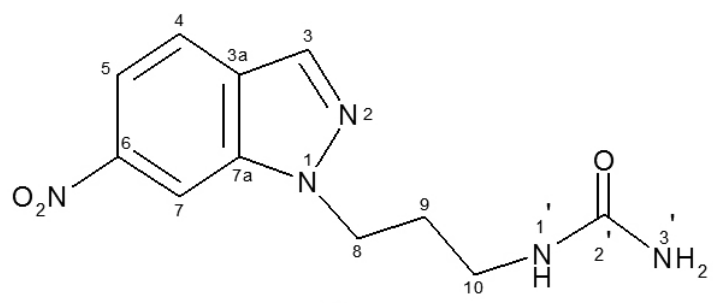

2

Figure 2: Structure of compound 2.

$\mathbf{N}^{1}$-\{3-(aminocarbamyl)-propyl\}-6-nitroindazole 2:

Yield: $74 \%$; m.p. $145-147^{\circ} \mathrm{C}$; IR $\left(\mathrm{cm}^{-1}\right): 752(\mathrm{C}-\mathrm{Cl}), 872(\mathrm{C}-\mathrm{N}), 1328(\mathrm{~N}-$ $\left.\mathrm{CH}_{2}\right), 1523\left(\mathrm{NO}_{2}\right), 1556(\mathrm{C}=\mathrm{C}), 1648(\mathrm{CO}), 1435,2839,2910\left(\mathrm{CH}_{2}\right), 3027$ $(\mathrm{CH}-\mathrm{Ar}), 3342(\mathrm{NH}), 3456\left(\mathrm{NH}_{2}\right) ;{ }^{1} \mathrm{H}$ NMR $\left(\mathrm{CDCl}_{3}, 300 \mathrm{MHz}\right) \delta: 2.18-2.22$ (m, 2H, H-9), 3.30-3.34 (m, 2H, H-10), 4.18 (t, 2H, J = $7.45 \mathrm{~Hz}, \mathrm{H}-8), 5.72$ (s, $1 \mathrm{H}, \mathrm{H}-1$ '), 5.92 (s, 2, H-3'), 7.34-7.96 (m, 4H, Ar-H); ${ }^{13} \mathrm{C}$ NMR (CDCl, 75

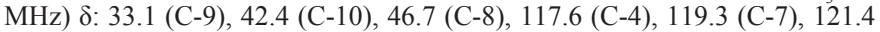
(C-5), 125.8 (C-3a), 134.5 (C-6), 136.7 (C-3), 139.2 (C-7a), 161.7 (C-2'); FAB-Mass $(\mathrm{m} / \mathrm{z}): 263\left[\mathrm{M}^{+}\right]$; Anal. Calcd for $\mathrm{C}_{11} \mathrm{H}_{13} \mathrm{~N}_{5} \mathrm{O}_{3}$ : C 50.18, H 4.97, N 26.60; found C 50.10, H 4.92, N 26.54.

General methods for the synthesis of compounds $3(\mathrm{a}-\mathrm{j})$

The compound 2 ( 0.026 mole) and benzaldehyde $(0.026$ mole) in ethanol $(100 \mathrm{ml})$ in the presence of 2-4 drops of glacial acetic acid were first stirred on a magnetic stirrer for about 2.00 hours followed by reflux on a steam bath for about 2.30 hours. The completion of the reaction was monitored by silica gel-G coated TLC plates. The product was filtered and cooled at room temperature. The filtered product was purified over a silica gel packed column chromatography using $\mathrm{CH}_{3} \mathrm{OH}: \mathrm{CHCl}_{3}(7: 3 \mathrm{v} / \mathrm{v})$ as eluant $(80 \mathrm{ml})$. The purified product was dried under vacuo and recrystallized from acetone at room temperature to furnish compound 3a (Figure 3).

Compounds 3 (b-j) have also been synthesized by using similar method as above.

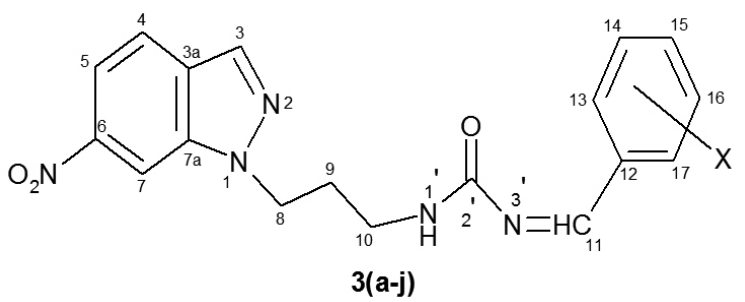

Figure 3: Structure of compound 3(a-j).

$N$-[3-(1H-6-nitroindazol-1-yl)-propyl]- $N$ '-[(phenyl)-methylidene]urea (3a):

Yield: $62 \%$; m.p. $147-148^{\circ} \mathrm{C}$; IR $\left(\mathrm{cm}^{-1}\right)$ : $742(\mathrm{C}-\mathrm{Cl}), 871(\mathrm{C}-\mathrm{N}), 1328(\mathrm{~N}-$ $\left.\mathrm{CH}_{2}\right), 1523\left(\mathrm{NO}_{2}\right), 1534(\mathrm{C}=\mathrm{C}), 1555(\mathrm{~N}=\mathrm{CH}), 1650(\mathrm{C}=\mathrm{O}), 1442,2839,2897$ $\left(\mathrm{CH}_{2}\right), 3027$ (CH-Ar), $3356(\mathrm{NH}) ;{ }^{1} \mathrm{H}$ NMR $\left(\mathrm{CDCl}_{3}, 300 \mathrm{MHz}\right) \delta: 2.22-2.27$ (m, 2H, H-9), 3.24-3.27 (m, 2H, H-10), 4.14 (t, 2H, J= $7.45 \mathrm{~Hz}, \mathrm{H}-8), 5.89$ (s, $\left.1 \mathrm{H}, \mathrm{H}-1^{\prime}\right), 7.98$ (s, 1H, H-11), 7.22-7.97 (m, 9H, Ar-H); ${ }^{13} \mathrm{C} \mathrm{NMR}\left(\mathrm{CDCl}_{3}, 75\right.$ MHz) $\delta: 28.1$ (C-9), 38.4 (C-10), 45.3 (C-8), 115.7 (C-4), 119.4 (C-7), 120.5 (C-5), 121.5 (C-3a), 121.8 (C-13 and C-17), 122.4 (C-14 and C-16), 126.5 (C15), 128.4 (C-12), 131.3 (C-6), 132.5 (C-3), 139.2 (C-7a), 150.6 (C-11), 159.9 (C-2'); FAB-Mass $(\mathrm{m} / \mathrm{z})$ : $351\left[\mathrm{M}^{+}\right]$; Anal. Calcd. for $\mathrm{C}_{18} \mathrm{H}_{17} \mathrm{~N}_{5} \mathrm{O}_{3}: \mathrm{C} 61.53, \mathrm{H}$ 4.87, N 19.93; found C 61.51, H 4.80, N 19.85 .

$N$-[3-(1 H-6-nitroindazol-1-yl)-propyl]- $N^{\prime}$-[(4-chlorophenyl)methylidene]-urea (3b):

Yield: $66 \%$; m.p. $165-167{ }^{\circ} \mathrm{C}$; IR $\left(\mathrm{cm}^{-1}\right)$ : $746(\mathrm{C}-\mathrm{Cl}), 905(\mathrm{C}-\mathrm{N}), 1351(\mathrm{~N}-$ $\left.\mathrm{CH}_{2}\right), 1534\left(\mathrm{NO}_{2}\right), 1572(\mathrm{C}=\mathrm{C}), 1580(\mathrm{~N}=\mathrm{CH}), 1633(\mathrm{C}=\mathrm{O}), 1447,2845,2917$ $\left(\mathrm{CH}_{2}\right), 3014(\mathrm{CH}-\mathrm{Ar}), 3344(\mathrm{NH}) ;{ }^{1} \mathrm{H}$ NMR $\left(\mathrm{CDCl}_{3}, 300 \mathrm{MHz}\right) \delta: 2.23-2.28$ (m, 2H, H-9), 3.40-3.45 (m, 2H, H-10), 4.26 (t, $2 \mathrm{H}, J=7.45 \mathrm{~Hz}, \mathrm{H}-8), 5.66$ (s,
1H, H-1') 7.89 (s, 1H, H-11), 7.71-8.16 (m, 8H, Ar-H); ${ }^{13} \mathrm{C} \mathrm{NMR} \mathrm{(CDCl}, 75$ MHz) $\delta: 28.6$ (C-9), 38.7 (C-10), 46.6 (C-8), 118.5 (C-4), 121.4 (C-7), 122.4 (C-5), 124.7 (C-3a), 123.9 (C-13 and C-17), 124.2 (C-14 and C-16), 127.4 (C15), 129.5 (C-12), 132.1 (C-6), 133.5 (C-3), 141.9 (C-7a), 150.6 (C-11), 161.8 (C-2'); FAB-Mass (m/z): 385 [M+]; Anal. Calcd. for $\mathrm{C}_{18} \mathrm{H}_{16} \mathrm{~N}_{5} \mathrm{O}_{3} \mathrm{Cl}$ : C 56.03, H 4.18, N 18.15; found C 55.97, H 4.15, N 18.12 .

$N$-[3-(1H-6-nitroindazol-1-yl)-propyl]- $N^{\prime}$-[(3-chlorophenyl)methylidenel-urea (3c):

Yield: $65 \%$; m.p. $162-163{ }^{\circ} \mathrm{C}$; IR $\left(\mathrm{cm}^{-1}\right): 751(\mathrm{C}-\mathrm{Cl}), 875(\mathrm{C}-\mathrm{N}), 1337(\mathrm{~N}-$ $\left.\mathrm{CH}_{2}\right), 1532\left(\mathrm{NO}_{2}\right), 1543(\mathrm{C}=\mathrm{C}), 1556(\mathrm{~N}=\mathrm{CH}), 1657(\mathrm{C}=\mathrm{O}), 1431,2848,2890$ $\left(\mathrm{CH}_{2}\right), 3032(\mathrm{CH}-\mathrm{Ar}), 3362(\mathrm{NH}) ;{ }^{1} \mathrm{H}$ NMR $\left(\mathrm{CDCl}_{3}, 300 \mathrm{MHz}\right) \delta: 2.21-2.27$ (m, 2H, H-9), 3.40-3.45 (m, 2H, H-10), $4.20(\mathrm{t}, 2 \mathrm{H}, J=7.45 \mathrm{~Hz}, \mathrm{H}-8), 5.62(\mathrm{~s}$, $1 \mathrm{H}, \mathrm{H}-1$ ') 7.90 (s, 1H, H-11), 7.75-8.20 (m, 8H, Ar-H); ${ }^{13} \mathrm{C} \mathrm{NMR} \mathrm{(CDCl,} 75$ MHz) $\delta: 31.4$ (C-9), 42.5 (C-10), 48.9 (C-8), 118.9 (C-4), 120.2 (C-7), 122.7 (C-5), 123.1 (C-3a), 123.5 (C-13), 123.9 (C-17), 125.6 (C-14), 126.3 (C-16), 129.6 (C-15), 131.4 (C-12), 133.9 (C-6), 134.4 (C-3), 141.7 (C-7a), 152.5 (C11), 162.7 (C-2'); FAB-Mass (m/z): 385 [M+']; Anal. Calcd. for $\mathrm{C}_{18} \mathrm{H}_{16} \mathrm{~N}_{5} \mathrm{O}_{3} \mathrm{Cl}$ : C 56.03, H 4.18, N 18.15; found C 55.99, H 4.17, N,18.11.

$N$-[3-(1 H-6-nitroindazol-1-yl)-propyl]- $N$ '-[(2-chlorophenyl)methylidene]-urea (3d):

Yield: $62 \%$; m.p. $164-165^{\circ} \mathrm{C}$; IR $\left(\mathrm{cm}^{-1}\right): 746(\mathrm{C}-\mathrm{Cl}), 878(\mathrm{C}-\mathrm{N}), 1344(\mathrm{~N}-$ $\left.\mathrm{CH}_{2}\right), 1529\left(\mathrm{NO}_{2}\right), 1537(\mathrm{C}=\mathrm{C}), 1566(\mathrm{~N}=\mathrm{CH}), 1661(\mathrm{C}=\mathrm{O}), 1453,2852,2892$ $\left(\mathrm{CH}_{2}\right), 3034(\mathrm{CH}-\mathrm{Ar}), 3360(\mathrm{NH}) ;{ }^{1} \mathrm{H}$ NMR $\left(\mathrm{CDCl}_{3}, 300 \mathrm{MHz}\right) \delta: 2.31-2.35$ (m, 2H, H-9), 3.40-3.47 (m, 2H, H-10), 4.21 (t, $2 \mathrm{H}, J=7.40 \mathrm{~Hz}, \mathrm{H}-8), 5.64$ (s,

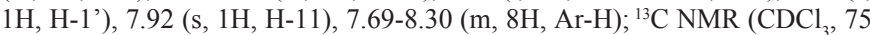
MHz) $\delta: ~ 31.7$ (C-9), 42.8 (C-10), 48.4 (C-8), 117.8 (C-4), 119.7 (C-7), 123.3 (C-5), 123.6 (C-3a), 123.9 (C-13), 124.0 (C-17), 124.6 (C-14), 124.8 (C-16), 126.6 (C-15), 130.2 (C-12), 133.8 (C-6), 134.5 (C-3), 140.1 (C-7a), 152.7 (C11), 160.7 (C-2'); Mass(FAB): $385\left[\mathrm{M}^{+}\right]$; Anal. Calcd. for $\mathrm{C}_{18} \mathrm{H}_{16} \mathrm{~N}_{5} \mathrm{O}_{3} \mathrm{Cl}$ : C 56.03, H 4.18, N 18.15; found C 55.97, H 4.15, N 18.14

$\mathrm{N}$-[3-(1 H-6-nitroindazol-1-yl)-propyl]- $N^{\prime}$-[(4-bromophenyl)methylidene]-urea (3e):

Yield: $64 \%$; m.p. $159-161{ }^{\circ} \mathrm{C}$; IR $\left(\mathrm{cm}^{-1}\right): 749(\mathrm{C}-\mathrm{Cl}), 881(\mathrm{C}-\mathrm{N}), 1340(\mathrm{~N}-$ $\left.\mathrm{CH}_{2}\right), 1526\left(\mathrm{NO}_{2}\right), 1538(\mathrm{C}=\mathrm{C}), 1565(\mathrm{~N}=\mathrm{CH}), 1656(\mathrm{C}=\mathrm{O}), 1454,2847,2900$ $\left(\mathrm{CH}^{2}\right), 3035$ (CH-Ar), $3367(\mathrm{NH}) ;{ }^{1} \mathrm{H}$ NMR $\left(\mathrm{CDCl}_{3}, 300 \mathrm{MHz}\right) \delta: 2.30-2.36$ (m, 2H, H-9), 3.41-3.50 (m, 2H, H-10), 4.33 (t, 2H, J=7.50 Hz, H-8), 5.65 (s, $\left.1 \mathrm{H}, \mathrm{H}-1^{\prime}\right), 7.93(\mathrm{~s}, 1 \mathrm{H}, \mathrm{H}-11), 7.74-8.26(\mathrm{~m}, 8 \mathrm{H}, \mathrm{Ar}-\mathrm{H}) ;{ }^{13} \mathrm{C} \mathrm{NMR}\left(\mathrm{CDCl}_{3}, 75\right.$

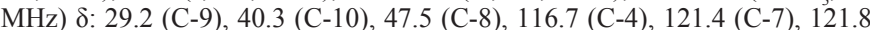
(C-57), 122.5 (C-13 and C-17), 122.9 (C-3a), 123.7 (C-14 and C-16), 127.3 (C15), 129.1 (C-12), 131.6 (C-6), 132.9 (C-3), 142.4 (C-7a), 153.7 (C-11), 160.0 (C-2'); FAB-Mass $(m / z)$ : 430 [M+ $]$; Anal. Calcd. for $\mathrm{C}_{18} \mathrm{H}_{16} \mathrm{~N}_{5} \mathrm{O}_{3} \mathrm{Br}$ : C 50.24, $\mathrm{H}$ 3.74, N 16.27; found C 50.21, H 3.71, N 16.22.

$\mathrm{N}$-[3-(1 H-6-nitroindazol-1-yl)-propyl]- $N^{\prime}$-[(3-bromophenyl)methylidene]-urea (3f):

Yield: $68 \%$; m.p. $157-158^{\circ} \mathrm{C}$; IR $\left(\mathrm{cm}^{-1}\right): 755(\mathrm{C}-\mathrm{Cl}), 874(\mathrm{C}-\mathrm{N}), 1334(\mathrm{~N}-$ $\left.\mathrm{CH}_{2}\right), 1532\left(\mathrm{NO}_{2}\right), 1541(\mathrm{C}=\mathrm{C}), 1570(\mathrm{~N}=\mathrm{CH}), 1662(\mathrm{C}=\mathrm{O}), 1445,2846,2891$ $\left(\mathrm{CH}_{2}\right), 3042(\mathrm{CH}-\mathrm{Ar}), 3365(\mathrm{NH}) ;{ }^{1} \mathrm{H}$ NMR $\left(\mathrm{CDCl}_{3}, 300 \mathrm{MHz}\right) \delta: 2.21-2.29$ $(\mathrm{m}, 2 \mathrm{H}, \mathrm{H}-9), 3.42-3.50(\mathrm{~m}, 2 \mathrm{H}, \mathrm{H}-10), 4.28(\mathrm{t}, 2 \mathrm{H}, J=7.45 \mathrm{~Hz}, \mathrm{H}-8), 5.59(\mathrm{~s}$,

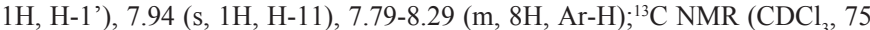
MHz) $\delta: 29.5$ (C-9), 32.7 (C-10), 46.3 (C-8), 117.9 (C-4), 120.4 (C-7), 121.5 (C-5), 123.1 (C-3a), 124.3 (C-13), 124.8 (C-17), 125.4 (C-14), 126.3 (C-16), 129.6 (C-15), 130.7 (C-12), 131.8 (C-6), 132.9 (C-3), 141.2 (C-7a), 154.6 (C11), 162.3 (C-2'); FAB-Mass $(\mathrm{m} / \mathrm{z})$ : $430\left[\mathrm{M}^{+}\right]$; Anal. Calcd. for $\mathrm{C}_{18} \mathrm{H}_{16} \mathrm{~N}_{5} \mathrm{O}_{3} \mathrm{Br}$ : C 50.24, H 3.74, N 16.27; found C 50.18, H 3.72, N 16.23.

$N$-[3-(1H-6-nitroindazol-1-yl)-propyl]- $N^{\prime}$-[(2-bromophenyl)methylidene]-urea (3g):

Yield: 69\%; m.p. 159-160 ${ }^{\circ} \mathrm{C}$; IR $\left(\mathrm{cm}^{-1}\right): 752(\mathrm{C}-\mathrm{Cl}), 876(\mathrm{C}-\mathrm{N}), 1335(\mathrm{~N}-$ $\left.\mathrm{CH}_{2}\right), 1531\left(\mathrm{NO}_{2}\right), 1544(\mathrm{C}=\mathrm{C}), 1567(\mathrm{~N}=\mathrm{CH}), 1654(\mathrm{C}=\mathrm{O}), 1446,2853,2893$ $\left(\mathrm{CH}_{2}\right), 3037$ (CH-Ar), $3370(\mathrm{NH}) ;{ }^{1} \mathrm{H}$ NMR $\left(\mathrm{CDCl}_{3}, 300 \mathrm{MHz}\right) \delta: 2.21-2.28$ (m, 2H, H-9), 3.50-3.55 (m, 2H, H-10), 4.29 (t, $2 \mathrm{H}, J=7.50 \mathrm{~Hz}, \mathrm{H}-8), 5.60(\mathrm{~s}$, $\left.1 \mathrm{H}, \mathrm{H}-1{ }^{\prime}\right), 7.97(\mathrm{~s}, 1 \mathrm{H}, \mathrm{H}-11), 7.81-8.25(\mathrm{~m}, 8 \mathrm{H}, \mathrm{Ar}-\mathrm{H}) ;{ }^{13} \mathrm{C} \mathrm{NMR}\left(\mathrm{CDCl}_{3}, 75\right.$ MHz) 8: 29.9 (C-9), 32.1 (C-10), 45.8 (C-8), 117.3 (C-4), 120.4 (C-7), 122.6 (C-5), 124.1 (C-3a), 124.5 (C-13), 124.9 (C-17), 125.6 (C-14), 126.1 (C-16), 129.9 (C-15), 131.4 (C-12), 134.3 (C-6), 135.4 (C-3), 142.6 (C-7a), 153.7 (C11), 159.5 (C-2'); FAB-Mass $(\mathrm{m} / \mathrm{z})$ : $430\left[\mathrm{M}^{+}\right]$; Anal. Calcd. for $\mathrm{C}_{18} \mathrm{H}_{16} \mathrm{~N}_{5} \mathrm{O}_{3} \mathrm{Br}$ : C 50.24, H 3.74, N 16.27; found C 50.18, H 3.70, N 16.21.

$N$-[3-(1H-6-nitroindazol-1-yl)-propyl]- $N^{\prime}$-[(4-nitrophenyl)methylidene]-urea $(3 \mathbf{h})$ :

Yield: $62 \%$; m.p. $162-163{ }^{\circ} \mathrm{C}$; IR $\left(\mathrm{cm}^{-1}\right): 750(\mathrm{C}-\mathrm{Cl}), 877(\mathrm{C}-\mathrm{N}), 1339(\mathrm{~N}-$ $\left.\mathrm{CH}_{2}\right), 1527\left(\mathrm{NO}_{2}\right), 1536(\mathrm{C}=\mathrm{C}), 1560(\mathrm{~N}=\mathrm{CH}), 1663(\mathrm{C}=\mathrm{O}), 1455,2845,2898$ $\left(\mathrm{CH}^{2}\right), 3038$ (CH-Ar), $3357(\mathrm{NH}) ;{ }^{1} \mathrm{H}$ NMR $\left(\mathrm{CDCl}_{3}, 300 \mathrm{MHz}\right) \delta: 2.24-2.30$ (m, 2H, H-9), 3.46-3.53 (m, 2H, H-10), 4.36 (t, $2 \mathrm{H}, J=7.40 \mathrm{~Hz}, \mathrm{H}-8), 5.61(\mathrm{~s}$, 1H, H-1'), 7.98 (s, 1H, H-11), 7.84-8.22 (m, 8H, Ar-H); ${ }^{13} \mathrm{C} \mathrm{NMR}\left(\mathrm{CDCl}_{3}, 75\right.$ 


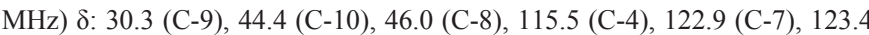
(C-5), 123.8 (C-3a), 124.3 (C-13 and C-17), 124.6 (C-14 and C-16), 128.4 (C15), 130.4 (C-12), 132.7 (C-6), 133.7 (C-3), 141.5 (C-7a), 154.6 (C-11), 161.9 (C-2'); FAB-Mass $(\mathrm{m} / \mathrm{z}): 396\left[\mathrm{M}^{+}\right]$; Anal. Calcd. for $\mathrm{C}_{18} \mathrm{H}_{16} \mathrm{~N}_{6} \mathrm{O}_{5}: \mathrm{C} 54.54, \mathrm{H}$ 4.06, N 21.20; found C 54.51, H 4.02, N 21.17.

$\mathrm{N}$-[3-(1 H-6-nitroindazol-1-yl)-propyl]- $N^{\prime}-[(3-n i t r o p h e n y l)-$ methylidene]-urea (3i):

Yield: $64 \%$; m.p. $166-167{ }^{\circ} \mathrm{C}$; IR $\left(\mathrm{cm}^{-1}\right)$ : $748(\mathrm{C}-\mathrm{Cl}), 882(\mathrm{C}-\mathrm{N}), 1336(\mathrm{~N}-$ $\left.\mathrm{CH}_{2}\right), 1535\left(\mathrm{NO}_{2}\right), 1540(\mathrm{C}=\mathrm{C}), 1563(\mathrm{~N}=\mathrm{CH}), 1658(\mathrm{C}=\mathrm{O}), 1456,2850,2896$ $\left(\mathrm{CH}_{2}\right), 3040(\mathrm{CH}-\mathrm{Ar}), 3358(\mathrm{NH}) ;{ }^{1} \mathrm{H}$ NMR $\left(\mathrm{CDCl}_{3}, 300 \mathrm{MHz}\right) \delta: 2.30-2.36$ (m, 2H, H-9), 3.50-3.54 (m, 2H, H-10), 4.37 (t, 2H, $J=7.40 \mathrm{~Hz}, \mathrm{H}-8), 5.63$ (s,

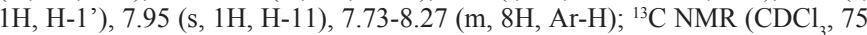

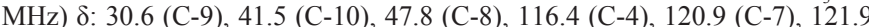
(C-5), 122.5 (C-3a), 123.4 (C-13), 123.7 (C-17), 124.8 (C-14), 125.7 (C-16), 128.1 (C-15), 131.5 (C-12), 133.9 (C-6), 135.6 (C-3), 140.6 (C-7a), 154.3 (C11), 161.9 (C-2'); FAB-Mass $(\mathrm{m} / \mathrm{z})$ : $396\left[\mathrm{M}^{+}\right]$; Anal. Calcd. for $\mathrm{C}_{18} \mathrm{H}_{16} \mathrm{~N}_{6} \mathrm{O}_{5}: \mathrm{C}$ 54.54, H 4.06, N 21.20; found C 54.49, H 4.03, N 21.16.

$N$-[3-(1 H-6-nitroindazol-1-yl)-propyl]- $N^{\prime}-[(2-n i t r o p h e n y l)-$ methylidene]-urea $(3 \mathbf{j})$ :

Yield: $66 \%$; m.p. $160-161{ }^{\circ} \mathrm{C}$; IR $\left(\mathrm{cm}^{-1}\right): 753(\mathrm{C}-\mathrm{Cl}), 879(\mathrm{C}-\mathrm{N}), 1338(\mathrm{~N}-$ $\left.\mathrm{CH}_{2}\right), 1526\left(\mathrm{NO}_{2}\right), 1542(\mathrm{C}=\mathrm{C}), 1557(\mathrm{~N}=\mathrm{CH}), 1660(\mathrm{C}=\mathrm{O}), 1447,2851,2901$ $\left(\mathrm{CH}_{2}\right), 3036(\mathrm{CH}-\mathrm{Ar}), 3369(\mathrm{NH}) ;{ }^{1} \mathrm{H}$ NMR $\left(\mathrm{CDCl}_{3}, 300 \mathrm{MHz}\right) \delta: 2.25-2.31$ (m, 2H, H-9), 3.42-3.49 (m, 2H, H-10), 4.25 (t, $2 \mathrm{H}, J=7.50 \mathrm{~Hz}, \mathrm{H}-8), 5.58$ (s, $1 \mathrm{H}, \mathrm{H}-1$ '), 7.93 (s, $1 \mathrm{H}, \mathrm{H}-11), 7.76-8.28(\mathrm{~m}, 8 \mathrm{H}, \mathrm{Ar}-\mathrm{H}) ;{ }^{13} \mathrm{C} \mathrm{NMR}\left(\mathrm{CDCl}_{3}, 75\right.$ MHz) $\delta: 30.8$ (C-9), 40.9 (C-10), 47.2 (C-8), 116.6 (C-4), 120.6 (C-7), 121.9 (C-5), 122.1 (C-13), 122.5 (C-3a), 122.9 (C-17), 123.5 (C-14), 123.9 (C-16), 127.2 (C-15), 128.6 (C-12), 131.6 (C-6), 132.9 (C-3), 139.7 (C-7a), 154.1 (C11), 162.2 (C-2'); FAB-Mass $(\mathrm{m} / \mathrm{z})$ : 396 [M+'] Anal. Calcd. for $\mathrm{C}_{18} \mathrm{H}_{16} \mathrm{~N}_{6} \mathrm{O}_{5}: \mathrm{C}$ 54.54, H 4.06, N 21.20; found C 54.47, H 4.04, N 21.12.

\section{General methods for the synthesis of compounds $4(\mathrm{a}-\mathrm{j})$ :}

The compound 3a $(0.011$ mole) and thioglycolic acid $(0.011$ mole $)$ in ethanol $(50 \mathrm{ml})$ in the presence of $\mathrm{ZnCl}_{2}$ were allowed to react at room temperature. The reaction mixture was first stirred on a magnetic stirrer for about 2.30 hours followed by reflux on a steam bath for about 5.00 hours. The completion of the reaction was monitored by silica gel-G coated TLC plates. The product was filtered and cooled at room temperature. The filtered product was purified over a silica gel packed column chromatography using $\mathrm{CH}_{3} \mathrm{OH}$ : $\mathrm{CHCl}_{3}(7: 3 \mathrm{v} / \mathrm{v})$ as eluant $(70 \mathrm{ml})$. The purified product was dried under vacuo and recrystallized from acetone at room temperature to furnish compound $4 \mathbf{a}$ (Figure 4). as above.

Compounds $\mathbf{4}(\mathbf{b}-\mathbf{j})$ have also been synthesized by using similar method

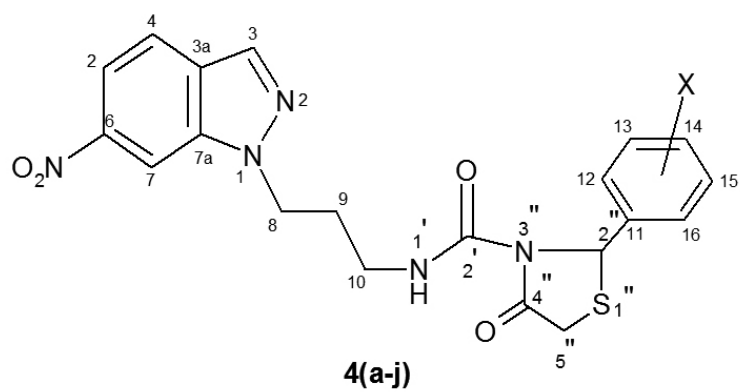

Figure 4: Structure of compound 4(a-j).

$N$-[3-(1 H-6-nitroindazol-1-yl)-propyl]-2-(phenyl)-4-oxo-1,3thiazolidine-carboxamide (4a):

Yield: $65 \%$; m.p. $160-161^{\circ} \mathrm{C}$; IR $\left(\mathrm{cm}^{-1}\right)$ : 663 (C-S-C), $880(\mathrm{C}-\mathrm{N}), 1339(\mathrm{~N}-$ $\left.\mathrm{CH}_{2}\right), 1531\left(\mathrm{NO}_{2}\right), 1545(\mathrm{C}=\mathrm{C}), 1661(\mathrm{C}=\mathrm{O}), 1737(\mathrm{CO}$ cyclic $), 1452,2848$, $2896\left(\mathrm{CH}_{2}\right), 2958\left(\mathrm{~S}-\mathrm{CH}_{2}\right), 3033,(\mathrm{CH}-\mathrm{Ar}), 3362(\mathrm{NH}){ }^{1} \mathrm{H}$ NMR $(\mathrm{CDCl}, 300$ MHz) $\delta$ : 2.26-2.32 (m, 2H, H-9), 3.28-3.33 (m, 2H, H-10), 3.40 (s, 2H, H-5"), $4.21(\mathrm{t}, 2 \mathrm{H}, J=7.45 \mathrm{~Hz}, \mathrm{H}-8), 5.21\left(\mathrm{~s}, 1 \mathrm{H}, \mathrm{H}-2^{\prime \prime}\right), 5.62$ (s, $\left.1 \mathrm{H}, \mathrm{H}-1{ }^{\prime}\right), 7.12-8.01$ (m, 9H, Ar-H); ${ }^{13} \mathrm{C}$ NMR (CDCl, 75 MHz) $\delta: 30.2$ (C-9), 33.5 (C-5”), 39.4 (C-10), 47.5 (C-8), 56.1 (C-2"), 117.3 (C-4), 122.5 (C-7), 123.3 (C-5), 128.6 (C-3a), 127.3 (C-12 and C-16), 129.7 (C-14), 130.1 (C-12 and C-15), 133.6 (C-6), 134.5 (C-3), 136.2 (C-11), 142.1 (C-7a), 161.6 (C-2'), 169.8 (C-4”); FAB-Mass $(\mathrm{m} / \mathrm{z})$ : $425\left[\mathrm{M}^{+}\right]$; Anal. Calcd. for $\mathrm{C}_{20} \mathrm{H}_{10} \mathrm{~N}_{5} \mathrm{O}$ S: C, 56.46, $\mathrm{H}, 4.50$, $\mathrm{N}, 16.46 \%$; found C, 56.41, H, 4.43, N, $16.39 \%$.

$\mathrm{N}$-[3-(1H-6-nitroindazol-1-yl)-propyl]-2-(4-chlorophenyl)-4-oxo-1,3-

\section{thiazolidine-carboxamide (4b):}

Yield: 67; m.p. 189-190 ${ }^{\circ} \mathrm{C}$; IR $\left(\mathrm{cm}^{-1}\right)$ : 667 (C-S-C), $882(\mathrm{C}-\mathrm{N}), 1345(\mathrm{~N}-$ $\left.\mathrm{CH}_{2}\right), 1532\left(\mathrm{NO}_{2}\right), 1550(\mathrm{C}=\mathrm{C}), 1662(\mathrm{C}=\mathrm{O}), 1742(\mathrm{CO}$ cyclic $), 1458,2851$, $2899\left(\mathrm{CH}_{2}\right), 2962\left(\mathrm{~S}-\mathrm{CH}_{2}\right), 3040(\mathrm{CH}-\mathrm{Ar}), 3366(\mathrm{NH}) ;{ }^{1} \mathrm{H}$ NMR $\left(\mathrm{CDCl}_{3}, 300\right.$ MHz) $\delta$ : 2.20-2.24 (m, 2H, H-9), 3.32 (s, 2H, H-5"), 3.35-3.39 (m, 2H, H-10), 4.28 (t, 2H, $J=7.45 \mathrm{~Hz}, \mathrm{H}-8), 5.23$ (s, 1H, H-2"), 5.72 (s, 1H, H-1'), 7.23-7.74 (m, 8H, Ar-H); ${ }^{13} \mathrm{C}$ NMR (CDCl, 75 MHz) $8: 33.8$ (C-9), 39.2 (C-5"), 46.6 (C-10), 50.9 (C-8), 62.4 (C-2"), 120.4 (C-4), 124.8 (C-7), 127.6 (C-5), 131.5 (C-3a), 132.3 (C-12 and C-16), 133.8 (C-14), 134.4 (C-13 and C-15), 136.7 (C-6), 139.7 (C-11), 140.9 (C-3), 144.9 (C-7a), 163.0 (C-2'), 171.7 (C-4"); FAB-Mass ( $\mathrm{m} / \mathrm{z}$ ): 460 [M $\mathrm{M}^{+}$; Anal. Calcd. for $\mathrm{C}_{20} \mathrm{H}_{18} \mathrm{~N}_{5} \mathrm{O}_{4} \mathrm{SCl}$ : C, 52.23, $\mathrm{H}, 3.94$, $\mathrm{N}, 15.22 \%$; found $\mathrm{C}, 52.19, \mathrm{H}, 3.89, \mathrm{~N}, 15.18 \%$.

$\mathrm{N}$-[3-(1H-6-nitroindazol-1-yl)-propyl]-2-(3-chlorophenyl)-4-0xo-1,3thiazolidine-carboxamide (4c):

Yield: $68 \%$; m.p. $185-186^{\circ} \mathrm{C}$; IR $\left(\mathrm{cm}^{-1}\right)$ : $671(\mathrm{C}-\mathrm{S}-\mathrm{C}), 884(\mathrm{C}-\mathrm{N}), 1341(\mathrm{~N}-$ $\left.\mathrm{CH}_{2}\right), 1536\left(\mathrm{NO}_{2}\right), 1552(\mathrm{C}=\mathrm{C}), 1665(\mathrm{C}=\mathrm{O}), 1745(\mathrm{CO}$ cyclic $), 1462,2853$, $2903\left(\mathrm{CH}_{2}\right), 2958\left(\mathrm{~S}-\mathrm{CH}_{2}\right), 3035(\mathrm{CH}-\mathrm{Ar}), 3365(\mathrm{NH}) ;{ }^{1} \mathrm{H}$ NMR $\left(\mathrm{CDCl}_{3}, 300\right.$ MHz) $\delta$ : 2.24-2.29 (m, 2H, H-9), 3.40 (s, 2H, H-5"), 3.43-3.48 (m, 2H, H-10), 4.22 (t, $2 \mathrm{H}, J=7.50 \mathrm{~Hz}, \mathrm{H}-8), 5.24$ (s, 1H, H-2"), 5.73 (s, 1H, H-1'), 7.30-7.80 (m, 8H, Ar-H); ${ }^{13} \mathrm{C}$ NMR (CDCl, $\left.75 \mathrm{MHz}\right) \delta: 31.1$ (C-9), 34.5 (C-5"), 43.8 (C-10), 50.9 (C-8), 60.1 (C-2”), 120.3 (C-4), 125.4 (C-7), 126.6 (C-5), 130.7 (C-3a), 131.2 (C-12), 131.9 (C-16), 133.4 (C-14), 134.6 (C-13), 135.2 (C-15), 137.3 (C-6), 137.9 (C-3), 139.4 (C-11), 142.7 (C-7a), 161.9 (C-2'), 172.5 (C4"); FAB-Mass ( $\mathrm{m} / \mathrm{z})$ : $460\left[\mathrm{M}^{+}\right]$; Anal. Calcd. for $\mathrm{C}_{20} \mathrm{H}_{18} \mathrm{~N}_{5} \mathrm{O}$ SCl: C, $52.23, \mathrm{H}$, 3.94, N, $15.22 \%$; found C, 52.17, H, 3.91, N, $15.19 \%$.

$\mathrm{N}$-[3-(1 H-6-nitroindazol-1-yl)-propyl]-2-(2-chloro phenyl)-4-ox0-1,3thiazolidine-carboxamide (4d):

Yield: $64 \%$; m.p. $180-181^{\circ} \mathrm{C}$; IR $\left(\mathrm{cm}^{-1}\right)$ : $672(\mathrm{C}-\mathrm{S}-\mathrm{C}), 885(\mathrm{C}-\mathrm{N}), 1342(\mathrm{~N}-$ $\left.\mathrm{CH}_{2}\right), 1540\left(\mathrm{NO}_{2}\right), 1547(\mathrm{C}=\mathrm{C}), 1670(\mathrm{C}=\mathrm{O}), 1749(\mathrm{CO}$ cyclic $), 1453,2849$, $2904\left(\mathrm{CH}_{2}\right), 2963\left(\mathrm{~S}_{-} \mathrm{CH}_{2}\right), 3036(\mathrm{CH}-\mathrm{Ar}), 3372(\mathrm{NH}) ;{ }^{1} \mathrm{H} \mathrm{NMR}\left(\mathrm{CDCl}_{3}, 300\right.$

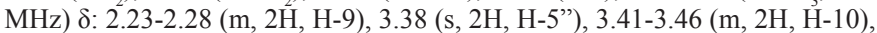
4.29 (t, 2H, $J=7.45 \mathrm{~Hz}, \mathrm{H}-8), 5.21$ (s, 1H, H-2"), 5.74 (s, 1H, H-1'), 7.36-7.86 (m, 8H, Ar-H): ${ }^{13} \mathrm{C}$ NMR (CDCl, $\left.75 \mathrm{MHz}\right) \delta: 31.6$ (C-9), 34.5 (C-5”), 42.7 (C-10), 50.4 (C-8), 60.9 (C-2"), 119.9 (C-4), 124.6 (C-7), 125.6 (C-5), 130.2 (C-3a), 130.7 (C-12), 131.1 (C-16), 132.6 (C-14), 133.8 (C-13), 134.6 (C-15), 135.5 (C-6), 136.4 (C-3), 138.1 (C-11), 139.9 (C-7a), 164.6 (C-2'), 172.7 (C4"); FAB-Mass $(\mathrm{m} / \mathrm{z})$ : $460\left[\mathrm{M}^{+}\right]$; Anal. Calcd. for $\mathrm{C}_{20} \mathrm{H}_{18} \mathrm{~N}_{5} \mathrm{O}_{4} \mathrm{SCl}$ : C, $52.23, \mathrm{H}$, $3.94, \mathrm{~N}, 15.22 \%$; found $\mathrm{C}, 52.22, \mathrm{H}, 3.90, \mathrm{~N}, 15.20 \%$.

$\mathrm{N}$-[3-(1 H-6-nitroindazol-1-yl)-propyl]-2-(4-bromo phenyl)-4-oxo-1,3thiazolidine-carboxamide (4e):

Yield: 65\%; m.p. $174-175^{\circ} \mathrm{C}$; IR ( $\left.\mathrm{cm}^{-1}\right): 670(\mathrm{C}-\mathrm{S}-\mathrm{C}), 886(\mathrm{C}-\mathrm{N}), 1340(\mathrm{~N}-$ $\left.\mathrm{CH}_{2}\right), 1542\left(\mathrm{NO}_{2}\right), 1555(\mathrm{C}=\mathrm{C}), 1671(\mathrm{C}=\mathrm{O}), 1750(\mathrm{CO}$ cyclic $), 1454,2855$, $2905\left(\mathrm{CH}_{2}\right), 2964\left(\mathrm{~S}_{-} \mathrm{CH}_{2}\right), 3038(\mathrm{CH}-\mathrm{Ar}), 3371(\mathrm{NH}) ;{ }^{1} \mathrm{H}$ NMR $\left(\mathrm{CDCl}_{3}, 300\right.$ MHz) $\delta: 2.27-2.34$ (m, 2H, H-9), 3.40 (s, 2H, H-5"), 3.42-3.47 (m, 2H, H-10), 4.31 (t, 2H, $J=7.45 \mathrm{~Hz}, \mathrm{H}-8), 5.22$ (s, 1H, H-2"), 5.79 (s, 1H, H-1'), 7.38-7.83 (m, 8H, Ar-H); ${ }^{13} \mathrm{C}$ NMR (CDCl, $\left.75 \mathrm{MHz}\right) \delta: 30.7$ (C-9), 35.4 (C-5"), 42.3 (C10), 49.1 (C-8), 59.6 (C-2"), 117.5 (C-4), 122.8 (C-7), 123.5 (C-5), 129.7 (C$3 \mathrm{a}), 130.5$ (C-12 and C-16), 132.3 (C-14), 133.7 (C-13 and C-15), 135.2 (C-6), 136.8 (C-3), 138.5 (C-11), 141.3 (C-7a), 162.6 (C-2'), 175.7 (C-4"); FAB-Mass $(\mathrm{m} / \mathrm{z}): 504\left[\mathrm{M}^{+}\right]$; Anal. Calcd. for $\mathrm{C}_{20} \mathrm{H}_{18} \mathrm{~N}_{5} \mathrm{O}_{4} \mathrm{SBr}$. C, 47.62, H, 3.52, N, 13.88 $\%$; found $\mathrm{C}, 47.57, \mathrm{H}, 3.45, \mathrm{~N}, 13.83 \%$.

$\mathrm{N}$-[3-(1H-6-nitroindazol-1-yl)-propyl]-2-(3-bromo phenyl)-4-oxo-1,3thiazolidine-carboxamide (4f):

Yield: $67 \%$; m.p. $178-179^{\circ} \mathrm{C}$; IR $\left(\mathrm{cm}^{-1}\right)$ : $665(\mathrm{C}-\mathrm{S}-\mathrm{C}), 888(\mathrm{C}-\mathrm{N}), 1346(\mathrm{~N}-$ $\left.\mathrm{CH}_{2}\right), 1534\left(\mathrm{NO}_{2}\right), 1556(\mathrm{C}=\mathrm{C}), 1664(\mathrm{C}=\mathrm{O}), 1738(\mathrm{CO}$ cyclic $), 1455,2860$, $2906\left(\mathrm{CH}_{2}\right), 2960\left(\mathrm{~S}-\mathrm{CH}_{2}\right), 3034(\mathrm{CH}-\mathrm{Ar}), 3365(\mathrm{NH}) ;{ }^{1} \mathrm{H}$ NMR $\left(\mathrm{CDCl}_{3}, 300\right.$ MHz) $\delta: 2.20-2.25$ (m, 2H, H-9), 3.28 (s, 2H, H-5"), 3.31-3.36 (m, 2H, H-10), 4.30 (t, $2 \mathrm{H}, J=7.50 \mathrm{~Hz}, \mathrm{H}-8), 5.20$ (s, 1H, H-2"), 5.80 (s, 1H, H-1'), 7.25-7.79 (m, 8H, Ar-H); ${ }^{13} \mathrm{C}$ NMR (CDCl, $\left.75 \mathrm{MHz}\right) \delta: 32.8$ (C-9), 35.7 (C-5"), 40.4 (C-10), 47.7 (C-8), 58.5 (C-2"), 117.8 (C-4), 123.4 (C-7), 124.3 (C-5), 129.8 (C-3a), 128.6 (C-12), 129.3 (C-16), 130.4 (C-14), 131.5 (C-13), 133.2 (C-6), 134.9 (C-3), 133.4 (C-15), 136.5 (C-11), 142.4 (C-7a), 165.3 (C-2'), 173.6 (C4"); FAB-Mass $(\mathrm{m} / \mathrm{z})$ : 504 [M+']; Anal. Calcd. for $\mathrm{C}_{20} \mathrm{H}_{18} \mathrm{~N}_{5} \mathrm{O}_{4} \mathrm{SBr}$ : C, 47.62, $\mathrm{H}$, $3.52, \mathrm{~N}, 13.88 \%$; found $\mathrm{C}, 47.61, \mathrm{H}, 3.45 \mathrm{~N}, 13.81 \%$.

$\mathrm{N}$-[3-(1 H-6-nitroindazol-1-yl)-propyl]-2-(2-bromo phenyl)-4-oxo-1,3thiazolidine-carboxamide $(4 \mathrm{~g})$ :

Yield: $68 \%$; m.p. $172-173^{\circ} \mathrm{C}$; IR $\left(\mathrm{cm}^{-1}\right)$ : $668(\mathrm{C}-\mathrm{S}-\mathrm{C}), 890(\mathrm{C}-\mathrm{N}), 1348(\mathrm{~N}-$ $\left.\mathrm{CH}_{2}\right), 1537\left(\mathrm{NO}_{2}\right), 1553(\mathrm{C}=\mathrm{C}), 1663(\mathrm{C}=\mathrm{O}), 1740(\mathrm{CO}$ cyclic $), 1461,2855$, $2910\left(\mathrm{CH}_{2}\right), 2963\left(\mathrm{~S}-\mathrm{CH}_{2}\right), 3041(\mathrm{CH}-\mathrm{Ar}), 3366(\mathrm{NH}) ;{ }^{1} \mathrm{H}$ NMR $\left(\mathrm{CDCl}_{3}, 300\right.$ MHz) $\delta$ : 2.22-2.26 (m, 2H, H-9), 3.35 (s, 2H, H-5"), 3.42-3.46 (m, 2H, H-10), 4.25 (t, $2 \mathrm{H}, J=7.40 \mathrm{~Hz}, \mathrm{H}-8), 5.19$ (s, 1H, H-2"), 5.77 (s, 1H, H-1'), 7.26-7.77 (m, 8H, Ar-H); ${ }^{13} \mathrm{C}$ NMR (CDCl, 75 MHz) $\delta: 34.4$ (C-9), 36.8 (C-5"), 39.9 (C-10), 48.4 (C-8), 59.5 (C-2”), 118.6 (C-4), 125.5 (C-7), 126.6 (C-5), 129.7 
(C-12), 130.2 (C-16), 130.5 (C-3a), 131.6 (C-14), 132.7 (C-13), 133.1 (C-15), 134.8 (C-6), 135.7 (C-3), 137.5 (C-11), 140.7 (C-7a), 165.8 (C-2'), 173.3 (C4"); FAB-Mass ( $\mathrm{m} / \mathrm{z}$ ): 504 [M+]; Anal. Calcd. for $\mathrm{C}_{20} \mathrm{H}_{18} \mathrm{~N}_{5} \mathrm{O}_{4} \mathrm{SBr}$ : C, 47.62, $\mathrm{H}$, $3.52, \mathrm{~N}, 13.88 \%$; found $\mathrm{C}, 47.59, \mathrm{H}, 3.48, \mathrm{~N}, 13.79 \%$.

$\mathrm{N}$-[3-(1 H-6-nitroindazol-1-yl)-propyl]-2-(4-nitrophenyl)-4-oxo-1,3thiazolidine-carboxamide (4h):

Yield: $66 \%$; m.p. $177-179^{\circ} \mathrm{C}$; IR $\left(\mathrm{cm}^{-1}\right): 673(\mathrm{C}-\mathrm{S}-\mathrm{C}), 893(\mathrm{C}-\mathrm{N}), 1349(\mathrm{~N}-$ $\left.\mathrm{CH}_{2}\right), 1538\left(\mathrm{NO}_{2}\right), 1549(\mathrm{C}=\mathrm{C}), 1666(\mathrm{C}=\mathrm{O}), 1746(\mathrm{CO}$ cyclic $), 1459,2854$, $2897\left(\mathrm{CH}_{2}\right), 2955\left(\mathrm{~S}-\mathrm{CH}_{2}\right), 3036(\mathrm{CH}-\mathrm{Ar}), 3368(\mathrm{NH}) ;{ }^{1} \mathrm{H}$ NMR $\left(\mathrm{CDCl}_{3}, 300\right.$ MHz) $\delta: 2.20-2.25$ (m, 2H, H-9), 3.39 (s, 2H, H-5"), 3.43-3.49 (m, 2H, H-10), 4.21 (t, $2 \mathrm{H}, J=7.45 \mathrm{~Hz}, \mathrm{H}-8), 5.36$ (s, 1H, H-2"), 5.70 (s, 1H, H-1'), 7.26-7.84 (m, 8H, Ar-H); ${ }^{13} \mathrm{C} \mathrm{NMR}\left(\mathrm{CDCl}_{3}, 75 \mathrm{MHz}\right) \delta: 32.2(\mathrm{C}-9), 36.9(\mathrm{C}-5$ "), $40.3(\mathrm{C}-$ 10), 51.8 (C-8), 57.2 (C-2"), 118.6 (C-4), 123.4 (C-5), 124.7 (C-7), 127.6 (C-12 and C-16), 128.8 (C-3a), 129.9 (C-14), 130.4 (C-13 and C-15), 135.7 (C-11), 132.6 (C-6), 133.7 (C-3), 143.1 (C-7a), 162.4 (C-2'), 174.3 (C-4"); FAB-Mass $(\mathrm{m} / \mathrm{z}): 470\left[\mathrm{M}^{+}\right]$; Anal. Calcd. for $\mathrm{C}_{20} \mathrm{H}_{18} \mathrm{~N}_{6} \mathrm{O}_{6} \mathrm{~S}: \mathrm{C}, 51.06, \mathrm{H}, 3.85, \mathrm{~N}, 17.86 \%$; found $\mathrm{C}, 51.00, \mathrm{H}, 3.78, \mathrm{~N}, 17.79 \%$.

$\mathrm{N}$-[3-(1H-6-nitroindazol-1-yl)-propyl]-2-(3-nitrophenyl)-4-0xo-1,3thiazolidine-carboxamide (4i):

Yield: $64 \%$; m.p. $180-181{ }^{\circ} \mathrm{C}$; IR $\left(\mathrm{cm}^{-1}\right)$ : $666(\mathrm{C}-\mathrm{S}-\mathrm{C}), 887(\mathrm{C}-\mathrm{N}), 1350(\mathrm{~N}-$ $\left.\mathrm{CH}_{2}\right), 1539\left(\mathrm{NO}_{2}\right), 1546(\mathrm{C}=\mathrm{C}), 1669(\mathrm{C}=\mathrm{O}), 1747$ (CO cyclic), 1456, 2850, $2905\left(\mathrm{CH}_{2}\right), 2957\left(\mathrm{~S}-\mathrm{CH}_{2}\right), 3037(\mathrm{CH}-\mathrm{Ar}), 3369(\mathrm{NH}) ;{ }^{1} \mathrm{H} \mathrm{NMR}\left(\mathrm{CDCl}_{3}, 300\right.$

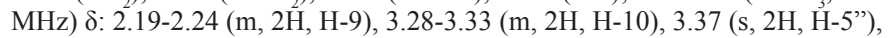
4.20 (t, $2 \mathrm{H}, J=7.40 \mathrm{~Hz}, \mathrm{H}-8), 5.41$ (s, $1 \mathrm{H}, \mathrm{H}-2$ ") 5.69 (s, $1 \mathrm{H}, \mathrm{H}-1$ '), $7.29-7.81$ (m, 8H, Ar-H); ${ }^{13} \mathrm{C}$ NMR ( $\left.\mathrm{CDCl}_{3}, 75 \mathrm{MHz}\right)$ 8: 33.4 (C-9), 37.4 (C-5"), 41.6 (C-10), 49.7 (C-8), 58.1 (C-2"), 119.6 (C-4), 124.8 (C-7), 125.6 (C-5), 128.4 (C-12), 129.5 (C-3a), 129.7 (C-16), 130.7 (C-14), 131.6 (C-13), 133.5 (C-6), 133.7 (C-15), 134.2 (C-3), 136.8 (C-11), 142.7 (C-7a), 163.4 (C-2'), 174.5 (C4"); FAB-Mass ( $\mathrm{m} / \mathrm{z})$ : $470\left[\mathrm{M}^{+}\right]$; Anal. Calcd. for $\mathrm{C}_{20} \mathrm{H}_{18} \mathrm{~N}_{6} \mathrm{O}_{6} \mathrm{~S}: \mathrm{C}, 51.06, \mathrm{H}$, $3.85, \mathrm{~N}, 17.86 \%$; found $\mathrm{C}, 51.04, \mathrm{H}, 3.80, \mathrm{~N}, 17.81 \%$.

$\mathrm{N}$-[3-(1H-6-nitroindazol-1-yl)-propyl]-2-(2-nitrophenyl)-4-oxo-1,3thiazolidine-carboxamide $(4 \mathrm{j})$ :

Yield: $68 \%$; m.p. $178-179^{\circ} \mathrm{C}$; IR $\left(\mathrm{cm}^{-1}\right): 675(\mathrm{C}-\mathrm{S}-\mathrm{C}), 889(\mathrm{C}-\mathrm{N}), 1347(\mathrm{~N}-$ $\left.\mathrm{CH}_{2}\right), 1541\left(\mathrm{NO}_{2}\right), 1548(\mathrm{C}=\mathrm{C}), 1668(\mathrm{C}=\mathrm{O}), 1748$ (CO cyclic), 1460, 2852, $2902\left(\mathrm{CH}_{2}\right), 2965\left(\mathrm{~S}-\mathrm{CH}_{2}\right), 3040(\mathrm{CH}-\mathrm{Ar}), 3370(\mathrm{NH}) ;{ }^{1} \mathrm{H}$ NMR $\left(\mathrm{CDCl}_{3}, 300\right.$ MHz) $\delta: 2.24-2.29$ (m, 2H, H-9), 3.29 (s, 2H, H-5"), 3.34-3.39 (m, 2H, H-10), $4.26(\mathrm{t}, 2 \mathrm{H}, J=7.50 \mathrm{~Hz}, \mathrm{H}-8), 5.22$ (s, $1 \mathrm{H}, \mathrm{H}-2$ "), 5.72 (s, 1H, H-1'), 7.34-7.92 (m, 8H, Ar-H); ${ }^{13} \mathrm{C}$ NMR (CDCl, $\left.75 \mathrm{MHz}\right) \delta: 34.7$ (C-9), 37.1 (C-5"), 41.5 (C-10), 48.6 (C-8), 60.4 (C-2"), 119.7 (C-4), 126.2 (C-7), 127.3 (C-5), 129.6 (C-12), 130.4 (C-3a), 130.7 (C-16), 131.3 (C-14), 132.8 (C-13), 134.1 (C-15), 135.3 (C-3), 136.9 (C-6), 137.3 (C-11), 142.8 (C-7a), 163.1 (C-2'), 175.7 (C4"); FAB-Mass $(\mathrm{m} / \mathrm{z})$ : $470\left[\mathrm{M}^{+}\right]$; Anal. Calcd. for $\mathrm{C}_{20} \mathrm{H}_{18} \mathrm{~N}_{6} \mathrm{O}_{6} \mathrm{~S}: \mathrm{C}, 51.06, \mathrm{H}$, $3.85, \mathrm{~N}, 17.86 \%$; found $\mathrm{C}, 51.01, \mathrm{H}, 3.82, \mathrm{~N}, 17.85 \%$.

\section{General methods for the synthesis of compounds $5(\mathrm{a}-\mathrm{j})$ :}

The compound 4a $(0.007$ mole $)$ and benzaldehyde $(0.007$ mole $)$ in ethanol $(50 \mathrm{ml})$ in the presence of $\mathrm{CH}_{3} \mathrm{CH}_{2} \mathrm{ONa}$ were allowed to react at room temperature. The reaction mixture was first stirred on a magnetic stirrer for about 2.00 hours followed by reflux on a steam bath for about 4.30 hours. The completion of the reaction was monitored by silica gel-G coated TLC plates. The product was filtered and cooled at room temperature. The filtered product was purified over a silica gel packed column chromatography using $\mathrm{CH}_{3} \mathrm{OH}$ : $\mathrm{CHCl}_{3}(7: 3 \mathrm{v} / \mathrm{v})$ as eluant as eluant $(50 \mathrm{ml})$. The purified product was dried under vacuo and recrystallized from acetone at room temperature to furnish compound 5a (Figure 5). as above.

Compounds $\mathbf{5}(\mathbf{b}-\mathbf{j})$ have also been synthesized by using similar method<smiles>[X]c1ccc(/C=C2\[S+]C(c3ccccc3)C(=O)N2C(=O)N(C)CCCn2ncc3ccc([N+](=O)[O-])cc32)cc1</smiles>

Figure 5: Structure of compound 5(a-j).
$N$-[3-(1H-6-nitroindazol-1-yl)-propyl]-2-(phenyl)-4-oxo-5(benzylidene)-1,3-thiazolidine-carboxamide (5a):

Yield: $62 \%$; m.p. $162-163{ }^{\circ} \mathrm{C}$; IR $\left(\mathrm{cm}^{-1}\right): 670$ (C-S-C), $885(\mathrm{C}-\mathrm{N}), 1343$ $\left(\mathrm{N}-\mathrm{CH}_{2}\right), 1536\left(\mathrm{NO}_{2}\right), 1666(\mathrm{C}=\mathrm{O}), 1741(\mathrm{CO}$ cyclic $), 1457,2854,2902\left(\mathrm{CH}_{2}\right)$, $2941(\mathrm{C}=\mathrm{CH}), 3038(\mathrm{CH}-\mathrm{Ar}), 3366(\mathrm{NH}) ;{ }^{1} \mathrm{H}$ NMR $\left(\mathrm{CDCl}_{3}, 300 \mathrm{MHz}\right) \delta: 2.12$ 2.16 (m, 2H, H-9), 3.43-3.49 (m, 2H, H-10), 4.16 (t, $2 \mathrm{H}, J=7.40 \mathrm{~Hz}, \mathrm{H}-8)$, 5.10 (s, 1H, H-2"), 5.69 (s, 1H, H-1'), 6.52 (s, 1H, H-17), 7.77-8.31 (m, 14H, Ar-H) ${ }^{13} \mathrm{C}$ NMR (CDCl, 75 MHz) $\delta: 32.8$ (C-9), 40.1 (C-10), 54.3 (C-8), 60.5 (C-2"), 117.6 (C-4), 123.2 (C-7), 125.4 (C-5), 127.3 (C-19 and C-23), 128.1 (C-12 and C-16), 129.2 (C-3a), 129.7 (C-20 and C-22), 130.5 (C-14), 131.4 (C13 and C-15), 132.1 (C-18), 134.8 (C-6), 135.7 (C-3), 137.6 (C-11), 138.2 (C17), 140.7 (C-5"), 142.3 (C-7a), 162.0 (C-2'), 170.7 (C-4"); FAB-Mass ( $m / 2$ ): $513\left[\mathrm{M}^{+}\right]$; Anal. Calcd. for $\mathrm{C}_{27} \mathrm{H}_{23} \mathrm{~N}_{5} \mathrm{O}_{4} \mathrm{~S}: \mathrm{C}, 63.14, \mathrm{H}, 4.51, \mathrm{~N}, 13.63 \%$; found C, 63.12, H, 4.49, N, $13.57 \%$.

$\mathrm{N}$-[3-(1H-6-nitroindazol-1-yl)-propyl]-2-(4-chlorophenyl)-4-oxo-5-(4chloro benzylidene)-1,3-thiazolidine-carboxamide (5b):

Yield: $64 \%$; m.p. $179-180^{\circ} \mathrm{C}$; IR $\left(\mathrm{cm}^{-1}\right)$ : $672(\mathrm{C}-\mathrm{S}-\mathrm{C}), 744(\mathrm{C}-\mathrm{Cl}), 887(\mathrm{C}-$ $\mathrm{N}), 1345\left(\mathrm{~N}-\mathrm{CH}_{2}\right), 1544\left(\mathrm{NO}_{2}\right), 1566(\mathrm{C}=\mathrm{CH}), 1672(\mathrm{C}=\mathrm{O}), 1742(\mathrm{CO}$ cyclic $)$ 1462, 2861, $2906\left(\mathrm{CH}_{2}\right), 2947(\mathrm{C}=\mathrm{CH}), 3040(\mathrm{CH}-\mathrm{Ar}), 3370(\mathrm{NH}) ;{ }^{1} \mathrm{H}$ NMR $\left(\mathrm{CDCl}_{3}, 300 \mathrm{MHz}\right) \delta: 2.15-2.20(\mathrm{~m}, 2 \mathrm{H}, \mathrm{H}-9), 3.45-3.51$ (m, 2H, H-10), 4.20 (t, $2 \mathrm{H}, J=7.40 \mathrm{~Hz}, \mathrm{H}-8), 5.16$ (s, 1H, H-2"), 5.75 (s, 1H, H-1'), 6.57 (s, 1H, $\mathrm{H}-17), 7.79-8.37$ (m, 12H, Ar-H) ${ }^{13} \mathrm{C}$ NMR (CDCl, $\left.75 \mathrm{MHz}\right) \delta: 32$ (C-9), 40.6 (C-10), 54.9 (C-8), 60.7 (C-2"), 118.2 (C-4), 123.5 (C-7), 126.5 (C-5), 127.7 (C-19 and C-23), 128.4 (C-12 and C-16), 129.4 (C-3a), 129.8 (C-20 and C-22), 131.3 (C-14), 131.9 (C-13 and C-15), 132.6 (C-18), 136.4 (C-3), 134.4 (C-6), 137.6 (C-11), 141.2 (C-17), 142.3 (C-5"), 142.9 (C-7a), 162.5 (C-2'), 171.1 (C-4"); FAB-Mass (m/z): 582 [M"]; Anal. Calcd. for $\mathrm{C}_{27} \mathrm{H}_{21} \mathrm{~N}_{5} \mathrm{O}_{4} \mathrm{SCl}_{2}: \mathrm{C}, 55.67$, $\mathrm{H}, 3.63, \mathrm{~N}, 12.02 \%$; found $\mathrm{C}, 55.57, \mathrm{H}, 3.55, \mathrm{~N}, 11.95 \%$.

$\mathrm{N}$-[3-(1H-6-nitroindazol-1-yl)-propyl]-2-(3-chloro phenyl)-4-oxo-5-(3chloro benzylidene)-1,3-thiazolidine-carboxamide (5c):

Yield: $62 \%$; m.p. $175-176^{\circ} \mathrm{C}$; IR $\left(\mathrm{cm}^{-1}\right)$ : $670(\mathrm{C}-\mathrm{S}-\mathrm{C}), 749(\mathrm{C}-\mathrm{Cl}), 888(\mathrm{C}-$ $\mathrm{N}), 1346\left(\mathrm{~N}-\mathrm{CH}_{2}\right), 1530\left(\mathrm{NO}_{2}\right), 1578(\mathrm{C}=\mathrm{CH}) 1647(\mathrm{C}=\mathrm{O}), 1743(\mathrm{CO}$ cyclic $)$, 1450, 2839, $2923\left(\mathrm{CH}_{2}\right), 2946(\mathrm{C}=\mathrm{CH}), 3047(\mathrm{CH}-\mathrm{Ar}), 3362(\mathrm{NH}) ;{ }^{1} \mathrm{H}$ NMR $\left(\mathrm{CDCl}_{3}, 300 \mathrm{MHz}\right) \delta: 2.19-2.24$ (m, 2H, H-9), 3.46-3.50 (m, 2H, H-10), 4.15 (t, $2 \mathrm{H}, J=7.50 \mathrm{~Hz}, \mathrm{H}-8), 5.22$ (s, 1H, H-2"), $5.74(\mathrm{~s}, 1 \mathrm{H}, \mathrm{H}-1$ '), $6.65(\mathrm{~s}, 1 \mathrm{H}$, $\mathrm{H}-17), 7.85-8.39(\mathrm{~m}, 12 \mathrm{H}, \mathrm{Ar}-\mathrm{H}) ;{ }^{13} \mathrm{C}$ NMR $\left(\mathrm{CDCl}_{3}, 75 \mathrm{MHz}\right) \delta: 35.8(\mathrm{C}-9)$, 44.1 (C-10), 49.3 (C-8), 60.9 (C-2”), 122.6 (C-4), 124.2 (C-7), 128.4 (C-5), 128.7 (C-19), 128.9 (C-23), 129.1 (C-12), 129.8 (C-16), 130.2 (C-3a), 130.7 (C-20), 131.2 (C-22), 131.8 (C-14), 132.2 (C-13), 132.7 (C-15), 133.1 (C-18), 135.3 (C-6), 136.6 (C-3), 138.6 (C-11), 140.6 (C-17), 141.3 (C-7a), 143.7 (C-5"), 166.0 (C-2'), 173.4 (C-4"); Mass (FAB) 582 [M+]; Anal. Calcd. for $\mathrm{C}_{27} \mathrm{H}_{21} \mathrm{~N}_{5} \mathrm{O}_{4} \mathrm{SCl}_{2}: \mathrm{C}, 55.67, \mathrm{H}, 3.63, \mathrm{~N}, 12.02 \%$; found $\mathrm{C}, 55.59, \mathrm{H}, 3.60, \mathrm{~N}$, $11.98 \%$.

$\mathrm{N}$-[3-(1H-6-nitroindazol-1-yl)-propyl]-2-(2-chloro phenyl)-4-oxo-5-(2chloro benzylidene)-1,3-thiazolidine-carboxamide (5d):

Yield: $68 \%$; m.p. $172-173^{\circ} \mathrm{C}$; IR $\left(\mathrm{cm}^{-1}\right)$ : $676(\mathrm{C}-\mathrm{S}-\mathrm{C}), 741(\mathrm{C}-\mathrm{Cl}), 890(\mathrm{C}-$ $\mathrm{N}), 1347\left(\mathrm{~N}-\mathrm{CH}_{2}\right), 1541\left(\mathrm{NO}_{2}\right), 1568(\mathrm{C}=\mathrm{CH}), 1667(\mathrm{C}=\mathrm{O}), 1744(\mathrm{CO}$ cyclic $)$, 1464, 2856, $2903\left(\mathrm{CH}_{2}\right), 2949(\mathrm{C}=\mathrm{CH}), 3042(\mathrm{CH}-\mathrm{Ar}), 3372(\mathrm{NH}) ;{ }^{1} \mathrm{H}$ NMR $\left(\mathrm{CDCl}_{3}, 300 \mathrm{MHz}\right) \delta: 2.22-2.27$ (m, 2H, H-9), 3.54-3.59 (m, 2H, H-10), 4.22 (t, $2 \mathrm{H}, J=7.50 \mathrm{~Hz}, \mathrm{H}-8), 5.18$ (s, 1H, H-2"), 5.77 (s, 1H, H-1'), 6.67 (s, $1 \mathrm{H}$, $\mathrm{H}-17), 7.83-8.40(\mathrm{~m}, 12 \mathrm{H}, \mathrm{Ar}-\mathrm{H}) ;{ }^{13} \mathrm{C}$ NMR $\left(\mathrm{CDCl}_{3}, 75 \mathrm{MHz}\right) \delta: 36.5(\mathrm{C}-9)$, 43.4 (C-10), 50.0 (C-8), 57.3 (C-2"), 119.6 (C-4), 125.5 (C-7), 126.4 (C-5), 128.3 (C-19), 128.7 (C-23), 129.4 (C-12), 129.7 (C-16), 130.2 (C-3a), 130.9 (C-20), 131.1 (C-22), 131.7 (C-14), 132.4 (C-13), 132.8 (C-15), 133.6 (C-18), 135.8 (C-6), 137.7 (C-3), 139.6 (C-11), 141.5 (C-17), 141.7 (C-7a), 143.8 (C5"), 163.7 (C-2'), 171.8 (C-4"); FAB-Mass $(\mathrm{m} / \mathrm{z}): 582$ [M+]; Anal. Calcd. for $\mathrm{C}_{27} \mathrm{H}_{21} \mathrm{~N}_{5} \mathrm{O}_{4} \mathrm{SCl}_{2}: \mathrm{C}, 55.67, \mathrm{H}, 3.63, \mathrm{~N}, 12.02 \%$; found $\mathrm{C}, 55.64, \mathrm{H}, 3.57, \mathrm{~N}$, $12.00 \%$.

$\mathrm{N}$-[3-(1H-6-nitroindazol-1-yl)-propyl]-2-(4-bromo phenyl)-4-oxo-5-(4bromo benzylidene)-1,3-thiazolidine-carboxamide (5e):

Yield: $64 \%$; m.p. $169-171^{\circ} \mathrm{C}$; IR $\left(\mathrm{cm}^{-1}\right): 679$ (C-S-C), $891(\mathrm{C}-\mathrm{N}), 1352$ $\left(\mathrm{N}-\mathrm{CH}_{2}\right), 1542(\mathrm{NO}), 1572(\mathrm{C}=\mathrm{CH}), 1670(\mathrm{C}=\mathrm{O}), 1746(\mathrm{CO}$ cyclic $), 1465$, 2859, $2904\left(\mathrm{CH}_{2}\right), 2945(\mathrm{C}=\mathrm{CH}), 3044(\mathrm{CH}-\mathrm{Ar}), 3375(\mathrm{NH}) ;{ }^{1} \mathrm{H} \mathrm{NMR}\left(\mathrm{CDCl}_{3}\right.$, $300 \mathrm{MHz}) \delta: 2.23-2.28(\mathrm{~m}, 2 \mathrm{H}, \mathrm{H}-9), 3.49-3.55(\mathrm{~m}, 2 \mathrm{H}, \mathrm{H}-10), 4.27\left(\mathrm{t}, 2 \mathrm{H}, J^{3}=\right.$ $7.40 \mathrm{~Hz}, \mathrm{H}-8$ ), 5.15 (s, 1H, H-2"), 5.78 (s, 1H, H-1'), 6.68 (s, 1H, H-17), 7.81$8.32(\mathrm{~m}, 12 \mathrm{H}, \mathrm{Ar}-\mathrm{H}) ;{ }^{13} \mathrm{C}$ NMR $\left(\mathrm{CDCl}_{3}, 75 \mathrm{MHz}\right) \delta: 33.7$ (C-9), 41.6 (C-10), 51.3 (C-8), 61.7 (C-2"), 121.6 (C-4), 123.6 (C-7), 125.5 (C-5), 129.3 (C-19 and C-23), 130.3 (C-12 and C-16), 130.9 (C-3a), 131.7 (C-20 and C-22), 132.5 (C-14), 133.6 (C-13 and C-15), 134.5 (C-18), 136.6 (C-6), 138.7 (C-3), 139.7 (C-17), 140.4 (C-11), 141.6 (C-7a), 143.5 (C-5"), 163.8 (C-2'), 171.5 (C-4”); Mass (FAB) $671\left[\mathrm{M}^{+}\right]$; Anal. Calcd. for $\mathrm{C}_{27} \mathrm{H}_{21} \mathrm{~N}_{5} \mathrm{O}_{4} \mathrm{SBr}_{2}: \mathrm{C}, 48.30, \mathrm{H}, 3.15, \mathrm{~N}$, $10.43 \%$; found $\mathrm{C}, 48.21, \mathrm{H}, 3.14, \mathrm{~N}, 10.39 \%$.

$\mathrm{N}$-[3-(1H-6-nitroindazol-1-yl)-propyl]-2-(3-bromophenyl)-4-oxo-5-(3- 
bromo benzylidene)-1,3-thiazolidine-carboxamide (5f):

Yield: $61 \%$; m.p. $173-174^{\circ} \mathrm{C}$; IR $\left(\mathrm{cm}^{-1}\right)$ : $680(\mathrm{C}-\mathrm{S}-\mathrm{C}), 892(\mathrm{C}-\mathrm{N}), 1348(\mathrm{~N}-$ $\left.\mathrm{CH}_{2}\right), 1538\left(\mathrm{NO}_{2}\right), 1573(\mathrm{C}=\mathrm{CH}), 1671(\mathrm{C}=\mathrm{O}), 1748$ (CO cyclic), 1466, 2860, $2905\left(\mathrm{CH}_{2}\right), 2944(\mathrm{C}=\mathrm{CH}), 3046(\mathrm{CH}-\mathrm{Ar}), 3367(\mathrm{NH}) ;{ }^{1} \mathrm{H} \mathrm{NMR}\left(\mathrm{CDCl}_{3}, 300\right.$ MHz) $\delta: 2.22-2.29$ (m, 2H, H-9), 3.47-3.52 (m, 2H, H-10), 4.24 (t, 2H, $J=7.45$ $\mathrm{Hz}, \mathrm{H}-8$ ), 5.23 (s, 1H, H-2"), 5.78 (s, 1H, H-1'), 6.58 (s, 1H, H-17), 7.89-8.36 (m, 12H, Ar-H); ${ }^{13} \mathrm{C}$ NMR ( $\left.\mathrm{CDCl}_{3}, 75 \mathrm{MHz}\right) \delta: 35.6$ (C-9), 41.4 (C-10), 48.8 (C-8), 61.5 (C-2"), 119.4 (C-4), 125.5 (C-7), 127.4 (C-5), 129.8 (C-19), 130.0 (C-23), 130.3 (C-12), 130.7 (C-16), 131.4 (C-3a), 131.7 (C-20), 132.0 (C-22), 132.5 (C-14), 133.4 (C-13), 133.8 (C-15), 134.7 (C-18), 136.6 (C-6), 137.2 (C3), 138.3 (C-11), 140.6 (C-17), 141.6 (C-7a), 142.7 (C-5"), 164.6 (C-2'), 174.7 (C-4"); FAB-Mass (m/z): 671 [M+]; Anal. Calcd. for $\mathrm{C}_{27} \mathrm{H}_{21} \mathrm{~N}_{5} \mathrm{O}_{4} \mathrm{SBr}_{2}$ : C, 48.30, $\mathrm{H}, 3.15, \mathrm{~N}, 10.43 \%$; found $\mathrm{C}, 48.27, \mathrm{H}, 3.10, \mathrm{~N}, 10.40 \%$.

$\mathrm{N}$-[3-(1H-6-nitroindazol-1-yl)-propyl]-2-(2-bromophenyl)-4-oxo-5-(2bromo benzylidene)-1,3-thiazolidine-carboxamide (5g):

Yield: $63 \%$; m.p. $165-166^{\circ} \mathrm{C}$; IR $\left(\mathrm{cm}^{-1}\right)$ : $674(\mathrm{C}-\mathrm{S}-\mathrm{C}), 885(\mathrm{C}-\mathrm{N}), 1343(\mathrm{~N}-$ $\left.\mathrm{CH}_{2}\right), 1536\left(\mathrm{NO}_{2}\right), 1565(\mathrm{C}=\mathrm{CH}) 1666(\mathrm{C}=\mathrm{O}), 1741(\mathrm{CO}$ cyclic $), 1457,2854$ $2902\left(\mathrm{CH}_{2}\right), 2941(\mathrm{C}=\mathrm{CH}), 3038(\mathrm{CH}-\mathrm{Ar}), 3366(\mathrm{NH}) ;{ }^{1} \mathrm{H}$ NMR $\left(\mathrm{CDCl}_{3}, 300\right.$ MHz) $\delta: 2.21-2.28$ (m, 2H, H-9), 3.50-3.55 (m, 2H, H-10), 4.25 (t, 2H, $J=7.45$ $\mathrm{Hz}, \mathrm{H}-8$ ), 5.27 (s, 1H, H-2"), 5.81 (s, 1H, H-1'), 6.62 (s, 1H, H-17), 7.84-8.42 $(\mathrm{m}, 12 \mathrm{H}, \mathrm{Ar}-\mathrm{H}) ;{ }^{13} \mathrm{C}$ NMR $\left(\mathrm{CDCl}_{3}, 75 \mathrm{MHz}\right) \delta: 34.4$ (C-9), 42.4 (C-10), 49.9 (C-8), 59.4 (C-2"), 121.2 (C-4), 124.8 (C-7), 126.3 (C-5), 130.6 (C-19), 130.8 (C-23), 131.1 (C-12), 131.7 (C-16), 132.5 (C-3a), 132.8 (C-20), 133.4 (C-22), 133.8 (C-14), 134.4 (C-13), 134.8 (C-15), 135.5 (C-18), 137.7 (C-6), 138.9 (C3), 139.4 (C-11), 140.5 (C-17), 141.3 (C-7a), 142.8 (C-5"), 165.6 (C-2'), 174.6 (C-4"); FAB-Mass ( $\mathrm{m} / \mathrm{z})$ : 671 [M+]; Anal. Calcd. for $\mathrm{C}_{27} \mathrm{H}_{21} \mathrm{~N}_{5} \mathrm{O}_{4} \mathrm{SBr}_{2}$ : C, 48.30, $\mathrm{H}, 3.15, \mathrm{~N}, 10.43 \%$; found $\mathrm{C}, 48.23, \mathrm{H}, 3.13, \mathrm{~N}, 10.42 \%$.

$\mathrm{N}$-[3-(1H-6-nitroindazol-1-yl)-propyl]-2-(4-nitrophenyl)-4-oxo-5-(4nitro benzylidene)-1,3-thiazolidine-carboxamide (5h):

Yield: $66 \%$; m.p. $170-171^{\circ} \mathrm{C}$; IR $\left(\mathrm{cm}^{-1}\right)$ : $673(\mathrm{C}-\mathrm{S}-\mathrm{C}), 895(\mathrm{C}-\mathrm{N}), 1351$ $\left(\mathrm{N}-\mathrm{CH}_{2}\right), 1544(\mathrm{NO}), 1567(\mathrm{C}=\mathrm{CH}), 1675(\mathrm{C}=\mathrm{O}), 1747(\mathrm{CO}$ cyclic $), 1461$, 2855, $2911\left(\mathrm{CH}_{2}\right), 2952(\mathrm{C}=\mathrm{CH}), 3043(\mathrm{CH}-\mathrm{Ar}), 3370(\mathrm{NH}) ;{ }^{1} \mathrm{H}$ NMR: ${ }^{1} \mathrm{H}$ NMR (CDCl, $300 \mathrm{MHz}) \delta: 2.21-2.25$ (m, 2H, H-9), 3.50-3.54 (m, 2H, H-10), 4.31 (t, $2 \mathrm{H}, J=7.50 \mathrm{~Hz}, \mathrm{H}-8), 5.23$ (s, $1 \mathrm{H}, \mathrm{H}-2$ "), 5.82 (s, $1 \mathrm{H}, \mathrm{H}-1$ '), 6.64 (s, $1 \mathrm{H}, \mathrm{H}-17), 7.86-8.46(\mathrm{~m}, 12 \mathrm{H}, \mathrm{Ar}-\mathrm{H}) ;{ }^{13} \mathrm{C}$ NMR $\left(\mathrm{CDCl}_{3}, 75 \mathrm{MHz}\right) \delta: 36.3(\mathrm{C}-$ 9), 44.3 (C-10), 50.5 (C-8), 59.5 (C-2"), 120.2 (C-4), 126.7 (C-7), 128.4 (C-5), 130.1 (C-19 and C-23), 131.6 (C-12 and C-16), 132.2 (C-3a), 132.7 (C-20 and C-22), 133.5 (C-14), 134.5 (C-13 and C-15), 135.3 (C-18), 137.4 (C-6), 139.3 (C-3), 139.8 (C-11), 141.5 (C-17), 142.4 (C-7a), 144.7 (C-5"), 164.2 (C-2'), 174.4 (C-4"); FAB-Mass $(\mathrm{m} / \mathrm{z})$ : 603 [M+]; Anal. Calcd. for $\mathrm{C}_{27} \mathrm{H}_{21} \mathrm{~N}_{7} \mathrm{O}_{8} \mathrm{~S}$ : C, $53.72, \mathrm{H}, 3.50, \mathrm{~N}, 16.24 \%$; found $\mathrm{C}, 53.69, \mathrm{H}, 3.49, \mathrm{~N}, 16.20 \%$

$\mathrm{N}$-[3-(1H-6-nitroindazol-1-yl)-propyl]-2-(3-nitro phenyl)-4-oxo-5-(3nitro benzylidene)-1,3-thiazolidine-carboxamide (5i):

Yield: $65 \%$; m.p. $173-175^{\circ} \mathrm{C}$; IR $\left(\mathrm{cm}^{-1}\right): 675(\mathrm{C}-\mathrm{S}-\mathrm{C}), 896(\mathrm{C}-\mathrm{N}), 1349(\mathrm{~N}-$ $\left.\mathrm{CH}_{2}\right), 1546\left(\mathrm{NO}_{2}\right), 1571(\mathrm{C}=\mathrm{CH}), 1676(\mathrm{C}=\mathrm{O}), 1745(\mathrm{CO}$ cyclic $), 1460,2856$, $2912\left(\mathrm{CH}_{2}\right), 2943(\mathrm{C}=\mathrm{CH}), 3045(\mathrm{CH}-\mathrm{Ar}), 3369(\mathrm{NH}) ;{ }^{1} \mathrm{H} \mathrm{NMR}\left(\mathrm{CDCl}_{3}, 300\right.$ MHz) $\delta: 2.26-2.30$ (m, 2H, H-9), 3.54-3.59 (m, 2H, H-10), 4.28 (t, $2 \mathrm{H}, J=7.40$ $\mathrm{Hz}, \mathrm{H}-8), 5.26$ (s, 1H, H-2"), 5.75 (s, 1H, H-1'), 6.71 (s, 1H, H-17), 7.82-8.45 (m, 12H, Ar-H); ${ }^{13} \mathrm{C}$ NMR (CDCl, $\left.75 \mathrm{MHz}\right) \delta: 34.4$ (C-9), 43.5 (C-10), 52.6 (C-8), 58.2 (C-2"), 122.4 (C-4), 124.9 (C-7), 126.7 (C-5), 131.6 (C-19), 131.9 (C-23), 132.2 (C-12), 132.9 (C-16), 133.4 (C-20), 133.7 (C-3a), 133.9 (C-22), 134.1 (C-14), 135.0 (C-13), 135.7 (C-15), 136.9 (C-18), 138.8 (C-6), 139.5 (C3), 140.2 (C-11), 141.4 (C-17), 141.3 (C-7a), 143.5 (C-5"), 165.7 (C-2'), 172.1 (C-4"); FAB-Mass ( $\mathrm{m} / \mathrm{z})$ : 603 [M+]; Anal. Calcd. for $\mathrm{C}_{27} \mathrm{H}_{21} \mathrm{~N}_{7} \mathrm{O}_{8} \mathrm{~S}: \mathrm{C}, 53.72, \mathrm{H}$, $3.50, \mathrm{~N}, 16.24 \%$; found $\mathrm{C}, 53.67, \mathrm{H}, 3.47, \mathrm{~N}, 16.22 \%$.

$\mathrm{N}$-[3-(1H-6-nitroindazol-1-yl)-propyl]-2-(2-nitro phenyl)-4-oxo-5-(2nitro benzylidene)-1,3-thiazolidine-carboxamide (5j):

Yield: $64 \%$; m.p. $172-173^{\circ} \mathrm{C}$; IR $\left(\mathrm{cm}^{-1}\right)$ : $681(\mathrm{C}-\mathrm{S}-\mathrm{C}), 897(\mathrm{C}-\mathrm{N}), 1352(\mathrm{~N}-$ $\left.\mathrm{CH}_{2}\right), 1543\left(\mathrm{NO}_{2}\right), 1569(\mathrm{C}=\mathrm{CH}), 1677(\mathrm{C}=\mathrm{O}), 1749(\mathrm{CO}$ cyclic $), 1458,2858$, $2914\left(\mathrm{CH}_{2}\right), 295^{\circ}(\mathrm{C}=\mathrm{CH}), 3048(\mathrm{CH}-\mathrm{Ar}), 3374(\mathrm{NH}) ;{ }^{1} \mathrm{H}$ NMR $\left(\mathrm{CDCl}_{3}, 300\right.$ MHz) $\delta: 2.33-2.37$ (m, 2H, H-9), 3.51-3.56 (m, 2H, H-10), 4.23 (t, $2 \mathrm{H}, J=7.40$ $\mathrm{Hz}, \mathrm{H}-8), 5.21$ (s, 1H, H-2"), 5.72 (s, 1H, H-1'), 6.59 (s, 1H, H-17), 7.78-8.41 $(\mathrm{m}, 12 \mathrm{H}, \mathrm{Ar}-\mathrm{H}) ;{ }^{13} \mathrm{C}$ NMR $\left(\mathrm{CDCl}_{1}, 75 \mathrm{MHz}\right) \delta: 35.4$ (C-9), $42.5(\mathrm{C}-10), 52.6$ (C-8), 58.6 (C-2"), 120.6 (C-4), 125.8 (C-7), 127.4 (C-5), 131.1 (C-19), 131.6 (C-23), 132.6 (C-12), 132.9 (C-16), 133.2 (C-3a), 133.7 (C-20), 133.9 (C-22), 134.6 (C-14), 135.4 (C-13), 135.8 (C-15), 136.4 (C-18), 138.5 (C-6), 140.2 (C3), 141.7 (C-11), 140.3 (C-17), 141.8 (C-7a), 144.7 (C-5”), 163.3 (C-2'), 173.5 (C-4"); FAB-Mass ( $\mathrm{m} / \mathrm{z})$ : $603\left[\mathrm{M}^{+}\right]$; Anal. Calcd. for $\mathrm{C}_{27} \mathrm{H}_{21} \mathrm{~N}_{7} \mathrm{O}_{8} \mathrm{~S}: \mathrm{C}, 53.72, \mathrm{H}$, $3.50, \mathrm{~N}, 16.24 \%$; found C, $53.70, \mathrm{H}, 3.42, \mathrm{~N}, 16.18 \%$

\section{Biological study}

\section{Antibacterial antifungal and antitubercular activities}

Series of newly synthesized compounds were active against selected microorganisms. The minimal inhibition concentrations were determined using the filter paper disc diffusion method and the concentrations have been used in $\mu \mathrm{g} / \mathrm{mL}$. All the synthesized compounds have been screened in vitro for their antibacterial activity against $B$. subtilis, E. coli and $S$. aureus and antifungal activity against $A$. niger, A. flavus and $C$. albicans Standards for antibacterial and antifungal activities Streptomycin and Griseofulvin respectively were used. The antitubercular activity screened against the M. tuberculosis. For the antitubercular activity isoniazid and rifampicin were used as standard. Standards also screened under the similar conditions for comparison. Results are given in Table 1 .

\section{Antiinflammatory activities}

Carageenan induced rat paw oedema method was employed for evaluating the antiinflammatory activity of compounds at a dose $50 \mathrm{mg} / \mathrm{kg}$ bw in albino rats (weighing 80-110 gm, each group contain 5 animal) using phenylbutazone as a standard drug for comparison at a dose $30 \mathrm{mg} / \mathrm{kg}$ bw. The rate paw oedema was produced by the method of winter et al. The percentage inhibition of inflammation was calculated by applying Newbould formula. In vivo study has been approved institutional ethical committee, Dr. H.S. Gour University, Sagar. Results of compounds $\mathbf{1 , 2}, \mathbf{3}(\mathbf{a}-\mathbf{j}), \mathbf{4}(\mathbf{a}-\mathbf{j})$ and $\mathbf{5}(\mathbf{a}-\mathbf{j})$ were given in Table 2

\section{RESULTS AND DISCUSSION}

The reaction of 1-bromo-3-chloropropane with 6-nitroindazole was carried out in the methanol to afford compound $\mathbf{1}$. The spectroscopic analyses of compound 1 showed absorption peaks for $\mathrm{N}-\mathrm{CH}$ and $\mathrm{C}-\mathrm{Cl}$ at $13268 \mathrm{~cm}^{-1}$ and $768 \mathrm{~cm}^{-1}$ respectively in the IR spectrum. This fact also supported by the disappearance of $\mathrm{NH}$ absorption of the 6-nitroindazole. The compound $\mathbf{1}$ on the reaction with urea yielded compound 2 . In the spectroscopic analyses of compound $\mathbf{2}$ we found three absorption peaks in IR spectrum for $\mathrm{NH}, \mathrm{NH}_{2}$ and $\mathrm{CO}$ at 3342,3456 and $1648 \mathrm{~cm}^{-1}$ respectively while absorption of $\mathrm{C}-\mathrm{Cl}$ has been disappeared in the IR spectrum of compound $\mathbf{1}$. This fact was also supported by ${ }^{1} \mathrm{H}$ and ${ }^{13} \mathrm{C}$ NMR spectra because two signals appeared in the ${ }^{1} \mathrm{H}$ NMR spectrum for $\mathrm{NH}$ and $\mathrm{NH}_{2}$ at $\delta 5.72$ and $\delta 5.92 \mathrm{ppm}$ respectively. The formation of the compound $\mathbf{2}$ was fully supported by a $\mathrm{CO}$ group gives a signal at $\delta$ $161.7 \mathrm{ppm}$ in the ${ }^{13} \mathrm{C}$ NMR spectrum of the compound 2. All the facts together are strong evidence for the synthesis of compound 2 . Compound $\mathbf{2}$ give the condensation reaction with substituted benzaldehydes to yield compounds 3(aj). Structure confirmed by IR, ${ }^{1} \mathrm{H}$ NMR and ${ }^{13} \mathrm{C}$ NMR spectra of compounds 3(a-j). In the IR spectra an absorption found in the range of $1555-1580 \mathrm{~cm}^{-1}$ while a strong signal appeared in the range of $\delta$ 7.89-7.98 and $\delta$ 150.6-154.6 $\mathrm{ppm}$ in the ${ }^{1} \mathrm{H}$ NMR and ${ }^{13} \mathrm{C}$ NMR spectra of compounds $3(\mathrm{a}-\mathrm{j})$ respectively. The facts also supported by the disappearance of the signal of $\mathrm{NH}$ in the ${ }^{1} \mathrm{H}$ NMR spectra. The compounds $\mathbf{3}(\mathbf{a}-\mathbf{j})$ on reaction with thioglycolic acid in the presence of $\mathrm{ZnCl}_{2}$ gives the cycloaddition reaction and produced a five membered cyclic ring known as thiazolidinone ring, compounds $\mathbf{4}(\mathbf{a}-\mathbf{j})$. The compounds $\mathbf{4}(\mathbf{a}-\mathbf{j})$ showed a characteristic absorption of the cyclic carbonyl group in the range of $1737-1750 \mathrm{~cm}^{-1}$ in the IR spectra. The ${ }^{1} \mathrm{H}$ NMR spectra aroused our attention and clearly indicate the presence of the active methylene group in the thiazolidine ring in the range of $\delta 3.28-3.40 \mathrm{ppm}$. The ${ }^{13} \mathrm{C}$ NMR spectra of compounds $4(\mathrm{a}-\mathbf{j})$ also supported the fact that cyclic carbonyl group present and a signal appeared in the range of $\delta 169.8-175.7 \mathrm{ppm}$. These all fact also supported by the two evidences that are (a) disappearance of $\mathrm{N}=\mathrm{CH}$ proton and (b) appearance of $\mathrm{N}-\mathrm{CH}$ proton in the range of $\delta 5.19-5.41 \mathrm{ppm}$ in the ${ }^{1} \mathrm{H}$ NMR spectra of compounds $4(\mathrm{a}-\mathrm{j})$. The compounds $\mathbf{4}(\mathrm{a}-\mathrm{j})$ underwent the Knoevenagel condensation reaction with substituted benzaldehydes in the presence of $\mathrm{C}_{2} \mathrm{H}_{5} \mathrm{ONa}$ to afford the compounds $\mathbf{5}(\mathbf{a}-\mathbf{j})$. In the ${ }^{1} \mathrm{H}$ NMR spectra of the compounds $\mathbf{5}(\mathrm{a}-\mathrm{j})$, we found the disappearance of two methylene protons of compounds $4(\mathbf{a}-\mathbf{j})$ and an appearance of a new signal for $\mathrm{C}=\mathrm{CH}$ in the range of $\delta 6.52-6.71 \mathrm{ppm}$ in the ${ }^{1} \mathrm{H}$ NMR and two new signals for $\mathrm{C}=\mathrm{CH}$ and $\mathrm{C}=\mathrm{CH}$ appeared in the range of $\delta 138.2-141.5$ and $\delta 140.7-144.7 \mathrm{ppm}$ in the ${ }^{13} \mathrm{C}$ NMR spectra of the compounds $\mathbf{5}(\mathbf{a}-\mathbf{j})$. These all above facts clearly confirmed the synthesis of all final products.

The results of the all described activities (antibacterial, antifungal, antitubercular and antiinflammatory) were summarized in Tables 1 and 2 . The result of the antimicrobial screening data revealed that all the synthesized compounds showed considerable and varied activities against the selected microorganisms. Antimicrobial, antitubercular and anti-inflammatory activities data (as shown in Table 1 and 2) revealed that all the synthesized compounds have a structure activity relationship (SAR) because an activity of compounds varies with substitution. Nitro group containing compounds showed higher activity than chloro and bromo group containing compounds. Chloro and bromo derivatives also have higher activity than other rested compounds. On the basis of SAR, concluded that the activity of compounds depends on electron withdrawing nature of the substituted groups. The sequence of the activity is following 
Table 1: Antibacterial, antifungal and antitubercular activities of compounds 1, 2, 3(a-j), 4(a-j) and 5(a-j).

\begin{tabular}{|c|c|c|c|c|c|c|c|}
\hline \multirow{2}{*}{ Comp. } & \multicolumn{3}{|c|}{ Antibacterial activity } & \multicolumn{3}{|c|}{ Antifungal activity } & \multirow{2}{*}{$\begin{array}{c}\begin{array}{c}\text { Antitubercular } \\
\text { activity }\end{array} \\
\text { M. tuberculosis }\end{array}$} \\
\hline & B. subtilis & E. coli & S. aureus & A. niger & A. flavus & C. albicans & \\
\hline 1 & 12.25 & 17.25 & 16.50 & 14.5 & 18.5 & 22.50 & 11.25 \\
\hline 2 & 10.25 & 14.25 & 13.25 & 12.5 & 15.25 & 21.75 & 10.75 \\
\hline $3 a$ & 9.25 & 12.25 & 11.25 & 10.50 & 10.75 & 21.75 & 9.50 \\
\hline $3 b$ & 7.50 & 6.75 & 6.25 & 10.25 & 8.25 & 18.25 & 7.25 \\
\hline $3 c$ & 7.25 & 6.50 & 6.50 & 8.50 & 10.5 & 18.75 & 7.50 \\
\hline $3 d$ & 7.75 & 7.25 & 6.75 & 12.5 & 8.75 & 17.50 & 7.25 \\
\hline $3 \mathrm{e}$ & 7.50 & 7.25 & 7.75 & 9.25 & 10.50 & 18.50 & 8.25 \\
\hline $3 \mathrm{f}$ & 8.25 & 7.50 & 7.25 & 8.50 & 13.50 & 17.25 & 7.50 \\
\hline $3 g$ & 7.75 & 8.00 & 7.50 & 15.25 & 8.75 & 18.75 & 7.75 \\
\hline $3 \mathrm{~h}$ & 6.25 & 5.75 & 6.25 & 12.50 & 10.25 & 16.50 & 6.25 \\
\hline $3 i$ & 6.25 & 5.25 & 5.50 & 8.25 & 10.50 & 12.75 & 3.75 \\
\hline $3 \mathrm{j}$ & 6.75 & 4.75 & 6.25 & 9.25 & 8.25 & 13.75 & 4.25 \\
\hline $4 a$ & 8.25 & 6.25 & 9.25 & 12.50 & 10.75 & 19.75 & 7.50 \\
\hline $4 \mathrm{~b}$ & 6.50 & 5.25 & 5.25 & 10.75 & 12.25 & 15.25 & 4.75 \\
\hline $4 \mathrm{c}$ & 6.25 & 5.00 & 5.50 & 12.50 & 10.75 & 14.75 & 4.50 \\
\hline $4 d$ & 5.50 & 4.75 & 5.50 & 7.50 & 8.75 & 14.50 & 4.25 \\
\hline $4 \mathrm{e}$ & 6.50 & 5.25 & 5.75 & 10.25 & 9.50 & 17.50 & 5.25 \\
\hline $4 \mathrm{f}$ & 7.25 & 5.75 & 6.25 & 8.50 & 12.50 & 15.25 & 4.50 \\
\hline $4 \mathrm{~g}$ & 6.25 & 5.00 & 5.50 & 9.75 & 12.75 & 14.75 & 4.75 \\
\hline $4 \mathrm{~h}$ & 5.25 & 4.75 & 6.25 & 8.50 & 7.25 & 13.50 & 4.25 \\
\hline $4 \mathrm{i}$ & 5.25 & 3.25 & 6.50 & 7.50 & 8.50 & 14.50 & 4.25 \\
\hline $4 \mathrm{j}$ & 3.75 & 5.50 & 5.25 & 7.75 & 6.25 & 13.75 & 3.75 \\
\hline $5 \mathrm{a}$ & 9.25 & 7.25 & 6.50 & 15.5 & 15.5 & 17.50 & 6.25 \\
\hline $5 b$ & 3.75 & 6.25 & 6.25 & 8.5 & 9.25 & 12.75 & 3.25 \\
\hline $5 c$ & 3.25 & 2.00 & 3.25 & 6.50 & 7.75 & 12.75 & 1.50 \\
\hline $5 \mathrm{~d}$ & 3.50 & 2.25 & 2.25 & 7.25 & 8.25 & 12.25 & 2.25 \\
\hline $5 \mathrm{e}$ & 3.25 & 2.00 & 2.50 & 7.50 & 6.75 & 12.75 & 2.50 \\
\hline $5 f$ & 3.50 & 2.25 & 2.50 & 6.50 & 7.75 & 12.50 & 1.25 \\
\hline $5 \mathrm{~g}$ & 4.00 & 6.25 & 3.75 & 9.25 & 10.5 & 14.50 & 4.25 \\
\hline $5 \mathrm{~h}$ & 3.25 & 1.75 & 2.25 & 6.50 & 7.50 & 12.25 & 1.50 \\
\hline $5 \mathrm{i}$ & 3.25 & 2.00 & 2.50 & 6.25 & 7.25 & 12.75 & 1.75 \\
\hline $5 \mathrm{j}$ & 3.25 & 1.75 & 2.25 & 6.50 & 7.25 & 12.50 & 2.25 \\
\hline Streptomycin & 3.25 & 1.75 & 2.25 & - & - & - & - \\
\hline Griseofulvin & - & - & - & 6.25 & 8.75 & 12.50 & - \\
\hline
\end{tabular}

the mic values of standard streptomycin for all bacteria strain and griseofulvin for all fungi strain were in the range of $1.25-3.25$ and $625-12.5 \mu \mathrm{g} / \mathrm{ml}$ respectively.

isoniazid and rifampicin were used as standards, mic values were 1.25 and $2.50 \mu \mathrm{g} / \mathrm{ml}$ respectively.

Table 2: Antiinflammatory activity of compounds 1, 2, 3(a-j), 4(a-j) and 5(a-j).

\begin{tabular}{|c|c|c|c|}
\hline Compound Code & $\begin{array}{c}\text { Before carageenan administration } \\
\text { (mean } \pm \text { SEM) }\end{array}$ & $\begin{array}{c}\text { Total increase in paw volume after 5 } \\
\text { hours (mean } \pm \text { SEM) }\end{array}$ & $\begin{array}{c}\text { Percent } \\
\text { inhibition }\end{array}$ \\
\hline 1 & $0.61 \pm 0.01$ & $0.25 \pm 0.01$ & 21.87 \\
\hline 2 & $0.62 \pm 0.03$ & $0.24 \pm 0.03$ & 25.00 \\
\hline $3 \mathrm{a}$ & $0.64 \pm 0.02$ & $0.23 \pm 0.02$ & 28.12 \\
\hline $3 \mathrm{~b}$ & $0.65 \pm 0.01$ & $0.22 \pm 0.01$ & 31.25 \\
\hline $3 \mathrm{c}$ & $0.67 \pm 0.02$ & $0.22 \pm 0.02$ & 31.25 \\
\hline $3 \mathrm{~d}$ & $0.60 \pm 0.01$ & $0.21 \pm 0.03$ & 34.37 \\
\hline $3 \mathrm{e}$ & $0.61 \pm 0.03$ & $0.23 \pm 0.03$ & 28.12 \\
\hline $3 \mathrm{f}$ & $0.62 \pm 0.01$ & $0.24 \pm 0.02$ & 25.00 \\
\hline $3 \mathrm{~h}$ & $0.64 \pm 0.03$ & $0.23 \pm 0.01$ & 28.12 \\
\hline $3 \mathrm{i}$ & $0.66 \pm 0.01$ & $0.21 \pm 0.01$ & 34.37 \\
\hline
\end{tabular}




\begin{tabular}{|c|c|c|c|}
\hline $3 \mathrm{j}$ & $0.65 \pm 0.02$ & $0.21 \pm 0.03$ & 34.37 \\
\hline $4 \mathrm{a}$ & $0.63 \pm 0.01$ & $0.22 \pm 0.02$ & 31.25 \\
\hline $4 \mathrm{~b}$ & $0.65 \pm 0.03$ & $0.19 \pm 0.01$ & 40.62 \\
\hline $4 \mathrm{c}$ & $0.64 \pm 0.01$ & $0.18 \pm 0.03$ & 43.75 \\
\hline $4 \mathrm{~d}$ & $0.62 \pm 0.02$ & $0.19 \pm 0.03$ & 40.62 \\
\hline $4 \mathrm{e}$ & $0.61 \pm 0.03$ & $0.19 \pm 0.02$ & 30.62 \\
\hline $4 \mathrm{f}$ & $0.62 \pm 0.01$ & $0.20 \pm 0.0$ & 40.62 \\
\hline $4 \mathrm{~g}$ & $0.63 \pm 0.03$ & $0.19 \pm 0.02$ & 56.25 \\
\hline $4 \mathrm{~h}$ & $0.64 \pm 0.02$ & $0.14 \pm 0.03$ & 53.12 \\
\hline $4 \mathrm{i}$ & $0.65 \pm 0.01$ & $0.15 \pm 0.01$ & 56.25 \\
\hline $4 \mathrm{j}$ & $0.64 \pm 0.03$ & $0.16 \pm 0.02$ & 50.00 \\
\hline $5 \mathrm{a}$ & $0.60 \pm 0.02$ & $0.14 \pm 0.02$ & 56.25 \\
\hline $5 \mathrm{~b}$ & $0.64 \pm 0.02$ & $0.13 \pm 0.01$ & 59.38 \\
\hline $5 \mathrm{c}$ & $0.66 \pm 0.02$ & $0.13 \pm 0.02$ & 59.38 \\
\hline $5 \mathrm{~d}$ & $0.68 \pm 0.02$ & $0.13 \pm 0.02$ & 59.38 \\
\hline $5 \mathrm{e}$ & $0.66 \pm 0.03$ & $0.12 \pm 0.01$ & 62.50 \\
\hline $5 \mathrm{f}$ & $0.65 \pm 0.02$ & $0.14 \pm 0.01$ & 56.25 \\
\hline $5 \mathrm{~g}$ & $0.67 \pm 0.02$ & $0.12 \pm 0.01$ & 62.50 \\
\hline $5 \mathrm{~h}$ & $0.64 \pm 0.03$ & $0.10 \pm 0.03$ & 68.75 \\
\hline $5 \mathrm{i}$ & $0.65 \pm 0.02$ & $0.11 \pm 0.02$ & 65.63 \\
\hline $5 \mathrm{j}$ & $0.67 \pm 0.03$ & $0.32 \pm 0.01$ & - \\
\hline Control & $0.66 \pm 0.02$ & $0.08 \pm 0.02$ & 75.00 \\
\hline Standard phenylbutazone & $0.68 \pm 0.03$ & & \\
\hline
\end{tabular}

$$
\mathrm{NO}_{2}>\mathrm{Cl}>\mathrm{Br}>\mathrm{H}
$$

Results of antibacterial activity of the compounds $5 \mathrm{c}, 5 \mathrm{e}, 5 \mathrm{~h}, 5 \mathrm{i}$ and $5 \mathrm{j}$ showed good activity against $B$. subtilis and $E$. coli. Compounds $5 \mathrm{~d}, 5 \mathrm{~h}$ and $5 \mathrm{j}$ showed good activity against $S$. aureus. Incase of antifungal activity compounds $5 \mathrm{c}, 5 \mathrm{f}, 5 \mathrm{~h}, 5 \mathrm{i}$ and $5 \mathrm{j}$ showed good activity against $A$. niger and compounds $5 \mathrm{e}, 5 \mathrm{~h}$ and $5 \mathrm{j}$ showed good activity against $A$. flavus while none compounds showed significant antifungal activity against $C$. albicans. Comounds $5 \mathrm{c}, 5 \mathrm{f}, 5 \mathrm{~h}$ and $5 \mathrm{i}$ showed significant antitubercular activity and $5 \mathrm{f}, 5 \mathrm{~h}, 5 \mathrm{i}$ and $5 \mathrm{j}$ showed good anti-inflammatory activity in the series. Results showed compounds containing nitro group $(5 \mathrm{~h}, 5 \mathrm{i}, 5 \mathrm{j})$ displayed good activity against all strains then containing chloro or bromo group.

\section{CONCLUSIONS}

In this study a new series of compounds synthesis and characterized, gave satisfactory results. Synthesized compounds screened for their biological study which displayed moderate to good activity.

\section{ACKNOWLEDGEMENT}

The authors are thankful to SAIF, Central Drugs Research Institute, Lucknow (India) for providing spectral and analytical data of the compounds. We are also thankful to Head, Department of Chemistry, Dr. H. S. Gour, University, Sagar (India) for giving the facilities to carry out the work.

\section{REFERENCE}

1.- S. Berhe, A. Slupe, C. Luster, A. C. Jr.Henry, D. L. Warner, L. H. Zalkow, E. M. Burgess, N. M. Enwerem, O. Bakare, Bioorg. Med. Chem., 18, 134, (2010).
2.- K. W. Woods, J. P. Fischer, A. Claiborne, T. Li, S. A. Thomas, Gui-Dong Zhu, R. B. Diebold, X. Liu, Yan Shi, V. Klinghofer, E. K. Han, R. Guan, S. R. Magnone, E. F. Johnson, J. J. Bouska, A. M. Olson, R. de Jong, T. Oltersdorf, Y. Luo, S. H. Rosenberg, V. L. Girandaa, Q. Lia, Bioorg. Med. Chem., 14, 6832, (2006).

3.- G. A. Pinna, M. A. Pirisi, Jean-Mario Mussinu, G. Murineddu, G. Loriga, A. Pau, G. E. Grella, ll Farmaco, 58, 749, (2003).

4.- B. Cottyn, F. Acher, B. Ramassamy, L. Alvey, M. Lepoivre, Y. Frapart, D. Stuehr, D. Mansuy, J-L Boucher, D. Vichard, Bioorg. Med. Chem., 16, 5962, (2008).

5.- M. Doris, G. Ulf, T. Carsten, F. Hans, Brain Research, 78, 353, (1998).

6.- A. Gerpe, G. Aguirre, L. Boiani, H. Cerecetto, M. Gonzalez, C. Olea-Azar, C. Rigol, J. D. Maya, A. Morello, O. E. Piro, V. J. Aran, A. Azqueta, Cerain A. de Lopez, A. Monge, M. A. Rojas, G. Yaluff, Bioorg. Med. Chem, 14, 3467, (2006).

7.- X. Xua, X. Qian, L. Zhong, J. Fluorine Chemistry, 126, 297, (2005).

8.- W. Li, Y. Lu, Z. Wang, James T. Dalton, D. D. Miller, Bioorg. Med. Chem. Lett., 17, 4113, (2007).

9.- I. Vazzana, E. Terranovaa, F. Mattioli, F. Sparatorea, Arkivok, 5, 364, (2004).

10.- J. M. Orrling, M. R. Marzahn, H. Gutierrez-de-Teran, J. Aqvist, B. M. Dunn, M. Larhed, Bioorg. Med. Chem., 17, 5933, (2009).

11.- G. Li, X. Qian, J. Cui, Q. Huang, D. Cui, R. Zhang, F. Liu, J. Fluorine Chemistry, 127, 182, (2006).

12.- E. Ami, K. Nakahara, A. Sato, J.-T. Nguyen, K. Hidaka, Y. Hamada, S. Nakatani, T. Kimura, Y. Hayashia, Y. Kisoa, Bioorg. Med. Chem. Lett., 17, 4213, (2007). 